\title{
Abstractions and Automated Algorithms for Mixed Domain Finite Element Methods
}

\author{
CÉCILE DAVERSIN-CATTY, Simula Research Laboratory, Norway \\ CHRIS N. RICHARDSON, BP Institute, University of Cambridge, United Kingdom \\ ADA J. ELLINGSRUD and MARIE E. ROGNES, Simula Research Laboratory, Norway
}

Mixed dimensional partial differential equations (PDEs) are equations coupling unknown fields defined over domains of differing topological dimension. Such equations naturally arise in a wide range of scientific fields including geology, physiology, biology, and fracture mechanics. Mixed dimensional PDEs are also commonly encountered when imposing non-standard conditions over a subspace of lower dimension, e.g., through a Lagrange multiplier. In this article, we present general abstractions and algorithms for finite element discretizations of mixed domain and mixed dimensional PDEs of codimension up to one (i.e., $n$ D- $m$ D with $|n-m| \leqslant 1$ ). We introduce high-level mathematical software abstractions together with lower-level algorithms for expressing and efficiently solving such coupled systems. The concepts introduced here have also been implemented in the context of the FEniCS finite element software. We illustrate the new features through a range of examples, including a constrained Poisson problem, a set of Stokes-type flow models, and a model for ionic electrodiffusion.

CCS Concepts: • Mathematics of computing $\rightarrow$ Solvers; Partial differential equations; $\bullet$ Computing methodologies $\rightarrow$ Modeling methodologies;

Additional Key Words and Phrases: FEniCS project, mixed dimensional, mixed domains, mixed finite elements

\section{ACM Reference format:}

Cécile Daversin-Catty, Chris N. Richardson, Ada J. Ellingsrud, and Marie E. Rognes. 2021. Abstractions and Automated Algorithms for Mixed Domain Finite Element Methods. ACM Trans. Math. Softw. 47, 4, Article 31 (September 2021), 36 pages.

https://doi.org/10.1145/3471138

\section{INTRODUCTION}

Mixed dimensional partial differential equations (PDEs) are systems of differential equations coupling solution fields defined over domains of different topological dimensions. Problem settings that call for such equations are in abundance across the natural sciences [26, 48], in multi-physics problems [13, 49], and in mathematics [8, 29]. For instance, in geology, fluid flow through faults and fractures in rocks can be modelled via mixed dimensional PDEs posed on a

This project has received funding from the European Research Council (ERC) under the European Union's Horizon 2020 research and innovation programme under grant agreement 714892 (Waterscales).

Authors' addresses: C. Daversin-Catty, A. J. Ellingsrud, and M. E. Rognes, Simula Research Laboratory, P.O. Box 134, 1325, Lysaker, Norway; emails: \{cecile, ada, meg\}@simula.no; C. N. Richardson, BP Institute, University of Cambridge, Madingley Road, Cambridge, CB3 0EZ, United Kingdom; email: cnr12@cam.ac.uk.

Permission to make digital or hard copies of all or part of this work for personal or classroom use is granted without fee provided that copies are not made or distributed for profit or commercial advantage and that copies bear this notice and the full citation on the first page. Copyrights for components of this work owned by others than ACM must be honored. Abstracting with credit is permitted. To copy otherwise, or republish, to post on servers or to redistribute to lists, requires prior specific permission and/or a fee. Request permissions from permissions@acm.org.

(C) 2021 Association for Computing Machinery.

0098-3500/2021/09-ART31 \$15.00

https://doi.org/10.1145/3471138 


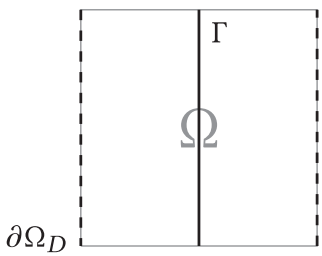

$$
\begin{array}{rlrl}
-\Delta u & =f & & \text { on } \Omega, \\
u=c & & \text { on } \Gamma, \\
u=0 & & \text { on } \partial \Omega_{D}, \\
\frac{\partial u}{\partial n}=0 & & \text { on } \partial \Omega \backslash \partial \Omega_{D} .
\end{array}
$$

Fig. 1. Find the solution $u: \Omega \rightarrow \mathbb{R}$ to the Poisson Equation (1) with mixed homogeneous boundary conditions on a two-dimensional unit square $\Omega=[0,1] \times[0,1] \subset \mathbb{R}^{2}$ such that $u$ is a constant $c$ along the topologically one-dimensional interior surface $\Gamma=\{(x, y) \mid x=0.5, y \in(0,1)\}$. To satisfy the latter constraint, introduce a Lagrange multiplier $\lambda: \Gamma \rightarrow \mathbb{R}$.

hierarchy of interacting domains of heterogeneous dimension $[9,45]$. In physiology, such equations can model blood flow in a three-dimensional lumen interacting with a topologically twodimensional elastic membrane i.e., the vessel wall [13]. Generally, Lagrange multipliers on lowerdimensional spaces are commonly used to impose non-standard boundary conditions or continuity properties over interfaces between subdomains [7, 48], see, e.g., Figure 1 for an idealized example.

For the numerical solution of mixed dimensional PDEs, the finite element method is a natural approach $[10,15,18]$. However, the efficient implementation of finite element discretizations for mixed dimensional PDEs is non-trivial-for a number of reasons. First, such discretizations involve manipulations of multiple meshes and submeshes of heterogeneous topological dimension. Second, the computation of local (element-wise) finite element tensors involve integrals of possibly restrictions of basis functions defined on cells of different dimensions. Third, the global assembly of the finite element matrices involve local-to-global mappings across different meshes and submeshes. And finally, the solution of the resulting linear systems requires efficient and appropriate linear algebra structures. As a result, the widespread application of mixed dimensional PDEs by domain specialists is hindered by a lack of numerical solution techniques and easy-to-use yet efficient software tools.

In view of the wide range of applications for mixed dimensional PDEs, a number of finite element software packages implement some mixed domain and mixed dimensional finite element features, including FreeFem++ [20], Feel++ [39], deal . II [6], or PorePy [25]. In particular, FreeFem++ [20] handles Lagrange multipliers in mixed and mortar methods defining finite element spaces on boundary meshes. deal. II [6] supports Lagrange multipliers on embedded, possibly non-matching, meshes, and in particular implements immersed finite elements methods [21]. Feel++ [39] also handles mixed dimensional problems defining trace meshes, used for example when implementing domain decomposition and mortar methods [43]. Finally, PorePy [25] implements mixed dimensional geometrical features providing an explicit representation of fractures with both finite volumes and virtual finite element discretizations. Still, the combination of a generic, automated and high-level software interface would allow for more rapid development of mixed dimensional discretizations and more widespread use.

Over the last 15 years, there has been a significant and growing interest in generic, highperformance finite element frameworks, as demonstrated by, e.g., the FEniCS Project [1, 19, 31], the Firedrake Project [40], Feel++ [39], FreeFEM [20], and NGSolve [44]. A shared design pattern is the combination of a high-level specification of the problem discretization, lower-level algorithms for problem solution, and automated code generation to bridge the gap between. This approach has been extremely successful, allowing for rapid development of advanced efficient numerical solvers for non-trivial PDEs and deployment by application scientists. In particular, FEniCS is organized as 
an open source collection of software components including the high-level domain-specific Unified Form Language (UFL) [2], the FEniCS Form Compiler (FFC) [32], and the problem solving environment DOLFIN [33, 34]. We refer to the above references for a more in-depth description of the FEniCS approach and components.

While FEniCS has offered native support for immersed manifolds since 2012 [42], support for discretizations of mixed domain- and mixed dimensional PDEs has been lacking in the core library. In response and driven by extensive user demand, several FEniCS extensions have been developed to remedy the situation. For instance, fenics_i i [27] implements the concept of trace spaces, while the multiphenics Python library [5] provides tools aiming to ease the prototyping of multiphysics problems. However, we argue that native support for mixed dimensional finite element methods within the core FEniCS framework is advantageous as it allows for, e.g., increased robustness in part due to more extensive testing and wider distribution, and easier development of auxiliary packages and techniques such as, e.g., the automated derivation of adjoint models [19]. Moreover, a formal description of the abstractions and algorithms involved in finite element methods for mixed dimensional PDEs is needed.

This work addresses and resolves the gap in available abstractions and algorithms, and importantly the formal description of such, for the automated numerical solution of mixed dimensional PDEs via finite element methods. In particular, we propose and advocate a light-weight design pattern for mixed dimensional finite element abstractions. This choice is discussed in more detail in Section 9.2. We revise and introduce new abstractions in the ULF for mixed function spaces, basis functions and integration domains allowing for coupled variational formulations defined over mixed domains. We also introduce a generalized assembly algorithm together with associated features such as submesh generation and block matrices. For the automated generation of local element tensor code from the symbolic representation, i.e., the form compilation, we present form component extraction algorithms and revised form compilation strategies.

The concepts and algorithms presented, here are implemented in UFL [2], FFC [32], and DOLFIN [33], are openly and freely available through the standard FEniCS distribution (development version). The source code reproducing the numerical results of Section 8 is available via [16].

The scope of this article is limited to mixed domain and mixed dimensional problems of codimension one at most, i.e., $n \mathrm{D}-m \mathrm{D}$ problems with $|n-m| \leqslant 1$ and to conforming meshes. Various techniques for handling non-matching meshes are discussed in the literature, such as, e.g., [12, 23], but not considered further here.

This article is organized as follows. In Section 2, we describe the mathematical scope of our mixed domain and mixed dimensional framework. We then address different aspects of the finite element method applied to mixed domain problems including key challenges in the subsequent sections. Section 3 is dedicated to meshes, nested submeshes, and mappings between them. The key features in UFL for defining and manipulating mixed domain function spaces and variational forms are introduced in Section 4. The local-to-global degree of freedom mapping is introduced in Section 5 as a key ingredient for the assembly of mixed domain and mixed dimensional variational forms. Abstract assembly algorithms, building on the construction of local element tensors and subsequent insertion using the local-to-global degree of freedom mappings, are detailed in Section 6. An overview of the revised FEniCS user interface and pipeline is given in Section 7 with emphasis on automated code generation of mixed domain and mixed dimensional local tensors and assembly features. Importantly, we present numerical results for various applications in Section 8 ranging from the idealized reference example introduced as Example 1 to more advanced models highlighting the relevance of our framework in biomedical applications. Section 10 provides some concluding remarks while discussing current limitations and future extensions. 


\section{MATHEMATICAL SCOPE AND CONCEPTS}

\subsection{Notation}

For convenience, we here provide an overview of the main notation used in this manuscript. In general, superscripts are used to indicate subdomain or block indices. In the text, all indices start at 1 . In the code, the corresponding indices start at 0 . The terms element and element-wise are used equivalently with cell or cell-wise, respectively.

$i, j$ : Indices associated with the number of subdomains.

$n, m$ : Indices associated with number of basis functions.

$r, s$ : Indices associated with form arity.

$|S|$ : Dimension of a finite set $S$.

$\Omega, \Omega^{i}$ : A domain, domain $i$ for $i=1, \ldots, I$ with $\Omega^{i} \subset \bar{\Omega}$.

$d^{i}$ : Topological dimension of $\Omega^{i}$.

$\mathbb{V}^{i}$ : Vector space relative to $\Omega^{i}$.

$\mathcal{T}, \mathcal{T}^{i}$ : A simplicial mesh with cells $K$, simplicial mesh of $\Omega^{i}$.

$\mathcal{S}(\mathcal{T}):$ The simplicial complex induced by the simplicial mesh $\mathcal{T}$.

$U^{i}$ : Finite element function space defined with respect to $\mathcal{T}^{i}$.

$N^{i}$ : Dimension of the finite element space $U^{i}: N^{i}=\operatorname{dim}\left(U^{i}\right)$.

$N_{K}^{i}$ : Dimension of the finite element space $U^{i}$ restricted to $K: N_{K}^{i}=\operatorname{dim}\left(\left.U^{i}\right|_{K}\right)$. If $K$ is a cell in $\mathcal{T}^{i}$, this is the local dimension of the finite element space $U^{i}$.

$\phi_{n}^{i}$ : Basis function for $U^{i}$ for $n=1, \ldots, N^{i}$.

$\mathcal{N}_{K}^{i}$ : A set of indices of basis functions in $U^{i}$ with $K$ in their support: $\mathcal{N}_{K}^{i}=\left\{n \in\left\{1, \ldots, N^{i}\right\} \mid K \subseteq \operatorname{supp}\left(\phi_{n}^{i}\right)\right\}$.

$\mathcal{V}, \mathcal{V}^{i}$ : Set of $n_{v}, n_{v}^{i}$ vertex indices in $\mathcal{T}, \mathcal{T}^{i}$.

$\mathcal{F}, \mathcal{F}^{i}$ : Set of $n_{f}, n_{f}^{i}$ facet indices in $\mathcal{T}, \mathcal{T}^{i}$.

$C, C^{i}$ : Set of $n_{c}, n_{c}^{i}$ cell indices in $\mathcal{T}, \mathcal{T}^{i}$.

$\mathcal{M}_{v}^{i}, \mathcal{M}^{i}$ : Child-to-parent vertex and cell index maps.

$\iota_{K}^{i}$ : Local-to-global degree of freedom map, $K \in \mathcal{T}^{i}$, for finite element space $U^{i}$.

$S_{K}$ : Star of $K$, defined as the set of cells in $\mathcal{T}$ containing $K$.

\subsection{Mixed Domains and Meshes}

We define a mixed domain PDE as a system of PDEs coupling fields $u^{i}: \Omega^{i} \rightarrow \mathbb{V}^{i}$ where $\Omega^{i} \subset \mathbb{R}^{d}$ is a bounded domain of geometrical dimension $d$ and topological dimension $d^{i}$, and $\mathbb{V}^{i}$ is a vector space for $i=1, \ldots, I$. We assume that there exists an $\Omega \subset \mathbb{R}^{d}$, a $d$-dimensional domain that embeds all the subdomains $\Omega^{i} \subseteq \Omega$, with $d \geqslant \max _{i} d^{i}$. We refer to $\Omega$ as the parent domain. We assume that $\Omega$ is polyhedral such that it admits a conforming discretization. The subdomains are assumed to be of codimension at most one relative to $\Omega$, i.e., $\left|d^{j}-d^{i}\right| \leqslant 1$ for all $i, j=1, \ldots, I$. We will use the term mixed dimensional PDE for a mixed domain PDE if there are $i, j$ such that $d^{i} \neq d^{j}$.

We assume that the parent domain $\Omega$ is partitioned by a mesh $\mathcal{T}$ consisting of a finite set of cells $\mathcal{T}=\{K\}$. For simplicity in terminology, we here consider the case of simplicial cells (intervals, triangles, and tetrahedra). Moreover, we assume that we can define a conforming mesh $\mathcal{T}^{i}$ of each subdomain $\Omega^{i}$, for $i=1, \ldots, I$, consisting of mesh entities (vertices, edges, faces, and cells) from $\mathcal{T}$. More precisely, we assume that $\Omega^{i}=\cup_{k}\left\{K_{k}^{i}\right\}$ where the submesh $\mathcal{T}^{i}=\left\{K_{k}^{i}\right\}_{k}$ consists of mesh entities $K_{k}^{i}$ from $\mathcal{T}$. In the language of complexes, let $S(\mathcal{T})$ be the simplicial complex defined by $\mathcal{T}$. By definition, $\Omega$ is then the underlying space of $S$. We assume that $\mathcal{T}$ and $\Omega^{i}$ for $i=1, \ldots, I$ are such that we can define simplicial meshes $\mathcal{T}^{i}$ with induced simplicial complexes $\mathcal{S}^{i}=\mathcal{S}\left(\mathcal{T}^{i}\right)$ such that $\Omega^{i}$ is the underlying space of $\mathcal{S}^{i}$ and such that $\mathcal{S}^{i}$ is a subcomplex of $\mathcal{S}$ for $i=1, \ldots, I$. 


\subsection{Finite Element Function Spaces}

We introduce function spaces $U^{i}$ for $i=1, \ldots, I$, each defined over $\Omega^{i}$, such that

$$
U^{i}=\left\{v^{i}: \Omega^{i} \rightarrow \mathbb{V}^{i}\right\}
$$

and assume that each unknown $u^{i} \in U^{i}$. The solution $u$ of a mixed domain PDE is hence an $I$-tuple $u=\left(u^{1}, \ldots, u^{I}\right)$ in the Cartesian product space $U$ :

$$
u \in U \equiv U^{1} \times U^{2} \times \cdots \times U^{I} .
$$

We refer to $U$ as a mixed function space with $U^{i}$ as subspaces.

We are here mainly concerned with finite element spaces $U^{i}$ defined relative to the submeshes $\mathcal{T}^{i}$ for $i=1, \ldots, I$. We assume that these discrete function spaces are indeed finite element spaces in the sense that the basis functions have localized support and can be defined element-wise. We write $N^{i}$ for the global dimension of the finite element space $U^{i}$, and $N_{K}^{i}$ for its local (element-wise) dimension i.e., $\operatorname{dim}\left(\left.U^{i}\right|_{K}\right)$ for $K \in \mathcal{T}^{i}$. We denote by $\left\{\phi_{n}^{i}\right\}_{n=1}^{N^{i}}$ the sets of basis functions spanning the discrete spaces $U^{i}$. Discrete solutions $u^{i} \in U^{i}$, for $i=1, \ldots, I$, can thus be expressed as a linear combination of these basis functions:

$$
u^{i}=\sum_{n=1}^{N^{i}} \bar{u}_{n}^{i} \phi_{n}^{i}
$$

with expansion coefficients (or, colloquially, degrees of freedom) $\bar{u}_{n}^{i}$ for $n=1, \ldots, N^{i}$. We emphasize the possibility of having different kinds of finite element spaces for the different function spaces. This is especially relevant for multiphysics problems for which the suitable function space properties can differ from one field to the other.

\subsection{Variational Forms and Formulations}

We consider discrete variational formulations of systems of linear or nonlinear PDEs and associated variational forms of arity $r \geq 0$. For time-dependent problems, we presuppose a time-stepping procedure yielding systems of PDEs at each time step. In general, we consider systems of PDEs that may be expressed in operator form with $I \in \mathbb{N}$ equations, each defined over $\Omega^{i}$ for $i=1, \ldots, I$. As our main emphasis is on finite element discretizations, we assume that a discrete variational formulation of the system is prescribed.

2.4.1 Linear Variational Problems. We first consider a general system of discrete linear variational equations: find $u \in U=U^{1} \times U^{2} \times \cdots \times U^{I}$ such that

$$
a^{i}\left(u, v^{i}\right)=L^{i}\left(v^{i}\right) \quad \forall v^{i} \in U^{i} \quad i=1, \ldots, I,
$$

where $a^{i}: U \times U^{i} \rightarrow \mathbb{R}$ is a bilinear form, $L^{i}: U^{i} \rightarrow \mathbb{R}$ is a linear form, and $U^{i}$ are appropriate finite element spaces defined over $\Omega^{i}$ and mapping into $\mathbb{V}^{i}$, for $i=1, \ldots, I$. To enhance readability, note that we present the case of coinciding trial and test subspaces here, however, we include numerical examples with differing test and trial spaces in Section 8.

By the linearity of $a^{i}$ and as the approximation space $U$ is defined as a Cartesian product of function spaces cf. (3), each bilinear form $a^{i}$ can be written as the sum of bilinear forms $a^{i, j}$ : $U^{j} \times U^{i} \rightarrow \mathbb{R}:$

$$
\exists a^{i, j} \mid a^{i}\left(u, v^{i}\right)=\sum_{j=1}^{I} a^{i, j}\left(u^{j}, v^{i}\right) \quad \forall i=1, \ldots, I .
$$

The discrete weak form of the whole coupled system for linear mixed problems thus consists of finding $u \in U$ such that

$$
a(u, v)=L(v) \quad \forall v \in U,
$$


with $v=\left(v^{1}, \ldots, v^{I}\right)$ and

$$
a(u, v)=\sum_{i=1}^{I} \sum_{j=1}^{I} a^{i, j}\left(u^{j}, v^{i}\right) \quad \text { and } \quad L(v)=\sum_{i=1}^{I} L^{i}\left(v^{i}\right) .
$$

In general, a variational form $a: U \times U \times \cdots \times U$ of arity $r$ can be decomposed into $r$ sums of arity- $r$ forms: $a^{i_{1}, i_{2}, \ldots, i_{r}}: U^{i_{r}} \times U^{i_{r-1}} \times \cdots \times U^{i_{1}}: \rightarrow \mathbb{R}:$

$$
a\left(u_{r}, u_{r-1}, \ldots, u_{1}\right)=\sum_{i_{1}=1}^{I} \cdots \sum_{i_{r}=1}^{I} a^{i_{1}, i_{2}, \ldots, i_{r}}\left(u_{r}^{i_{r}}, u_{r-1}^{i_{r-1}}, \ldots, u_{1}^{i_{1}}\right) .
$$

for $u_{s}=\left(u_{s}^{1}, u_{s}^{2}, \ldots, u_{s}^{I}\right)$ for $s=1, \ldots, r$. We will refer to $a^{i_{1}, i_{2}, \ldots, i_{r}}$ and specifically $a^{i, j}$ and $L^{i}$ as block forms. We will refer to a (block) form $a^{i_{s}, i_{s}, \ldots, i_{s}}$ for some $s \in\{1, \ldots, r\}$ as a diagonal (block) form.

The finite element solution of (7) typically involves the assembly of the bilinear form $a$ and linear form $L$, i.e., the construction of a matrix $A$ and a vector $L$ such that $u$ solves

$$
A \bar{u}=b,
$$

where $\bar{u}$ denotes the vector of expansion coefficients for the discrete field $u$ i.e.,

$$
\bar{u}=\left\{\bar{u}^{i}\right\}_{i=1}^{I}, \quad \bar{u}^{i}=\left\{\bar{u}_{n}^{i}\right\}_{n=1}^{N^{i}} \quad \forall i=1, \ldots, I .
$$

The resulting matrix $A$ and vector $b$ are logically block structured by construction, cf. (8). The actual ordering in an assembled system with entries

$$
\left[\begin{array}{c|c|c}
A^{1,1} & \cdots & A^{1, I} \\
\hline \vdots & \ddots & \vdots \\
\hline A^{I, 1} & \cdots & A^{I, I}
\end{array}\right]\left[\begin{array}{c}
\bar{u}^{1} \\
\hline \vdots \\
\hline \bar{u}^{I}
\end{array}\right]=\left[\begin{array}{c}
b^{1} \\
\hline \vdots \\
\hline b^{I}
\end{array}\right],
$$

would be a consequence of the choice of local-to-global maps. The diagonal blocks $A^{i, i}$ represent the uncoupled parts of the problem while the off-diagonal blocks $A^{i, j}$ for $i \neq j$ represent the interaction between fields living on any two subdomains $\Omega^{i}$ and $\Omega^{j}$. The elements of $A$ and $b$ are defined for $i, j=1, \ldots, I$ by

$$
A_{m, n}^{i, j}=a^{i, j}\left(\phi_{n}^{j}, \phi_{m}^{i}\right) \text { and } b_{m}^{i}=L^{i}\left(\phi_{m}^{i}\right), n=1, \ldots, N^{j}, m=1, \ldots, N^{i} .
$$

Example 1. To illustrate, we detail a variational formulation and the block structure of the mixed dimensional Poisson example introduced in Figure 1. As detailed in Section 2.2, we assume a mesh $\mathcal{T}$ of the parent domain $\Omega$ such that a subset of its facets induce a conforming submesh $\mathcal{T}^{2}$ of $\Gamma$. We identify $\Omega^{1}=\Omega, \mathcal{T}^{1}=\mathcal{T}$ and $\Omega^{2}=\Gamma$. Further, we let $U^{1} \subset H_{0}^{1}\left(\Omega^{1}\right)$ be a finite element space with zero trace on the Dirichlet boundary $\partial \Omega_{D}$ only, and let $U^{2}$ be a conforming finite element space of $L^{2}\left(\Omega^{2}\right)$. A discrete variational formulation describing (1) then reads: find $(u, \lambda) \in U \equiv U^{1} \times U^{2}$ such that

$$
\int_{\Omega} \nabla u \cdot \nabla v \mathrm{~d} x+\int_{\Gamma} \lambda v \mathrm{~d} s+\int_{\Gamma} \eta u \mathrm{~d} s=\int_{\Omega} f v \mathrm{~d} x+\int_{\Gamma} c \eta \mathrm{d} s,
$$

for all $(v, \eta) \in U^{1} \times U^{2}$.

The block decomposition (8) of the bilinear form $a(u, v)$ (resp. linear form $L(v)$ ) gives the subforms

$$
a^{1,1}(u, v)=\int_{\Omega} \nabla u \cdot \nabla v \mathrm{~d} x, \quad a^{1,2}(\lambda, v)=\int_{\Gamma} \lambda v \mathrm{~d} s, \quad a^{2,1}(u, \eta)=\int_{\Gamma} \eta u \mathrm{~d} s,
$$


with $a^{2,2}(\lambda, \eta)=0$, and

$$
L^{1}(v)=\int_{\Omega} f v \mathrm{~d} x, \quad L^{2}(\eta)=\int_{\Gamma} c \eta \mathrm{d} s .
$$

The block system corresponding to (14) then reads as follows (with $u^{1}=u$ and $u^{2}=\lambda$ ):

$$
\left[\begin{array}{c|c}
A^{1,1} & A^{1,2} \\
\hline A^{2,1} & A^{2,2}
\end{array}\right]\left[\begin{array}{c}
\bar{u}^{1} \\
\hline \bar{u}^{2}
\end{array}\right]=\left[\begin{array}{c}
b^{1} \\
\hline b^{2}
\end{array}\right],
$$

where the blocks $A^{i, j}$ and $b^{i}, i, j=1,2$ are obtained from (13).

2.4.2 Nonlinear Variational Problems. Nonlinear mixed domain problems lead to discrete variational formulations of the form: find $u \in U$ such that

$$
F^{i}\left(u ; v^{i}\right)=0 \quad \forall v^{i} \in U^{i},
$$

where the forms $F^{i}: U \times U^{i} \rightarrow \mathbb{R}$ may be nonlinear in $u \in U$ but are linear in the test functions $v^{i} \in U^{i}$ for $i=1, \ldots, I$. Combining the $I$ equations, the canonical nonlinear mixed formulation reads as: find $u \in U$ such that

$$
F(u ; v)=0 \quad \forall v \in U,
$$

with

$$
F(u ; v)=\sum_{i=1}^{I} F^{i}\left(u ; v^{i}\right) .
$$

Newton's method or variations are commonly used to solve such problems. Starting from an initial solution $u_{0}=\left(u_{0}^{1}, \ldots, u_{0}^{I}\right)$, each iteration solves the system

$$
J\left(u_{k} ; \cdot, v\right)\left(u_{k+1}-u_{k}\right)=-F\left(u_{k} ; v\right),
$$

where $J\left(u_{k} ; \cdot, v\right)$ is the Jacobian of $F\left(u_{k} ; v\right)$ at the $k$ th iteration. The discrete system at each iterate again has a block-shaped pattern

$$
\left[\begin{array}{c|c|c}
J^{1,1} & \cdots & J^{1, I} \\
\hline \vdots & \ddots & \vdots \\
\hline J^{I, 1} & \cdots & J^{I, I}
\end{array}\right]\left[\begin{array}{c}
\overline{\delta u^{0}} \\
\hline \vdots \\
\hline \overline{\delta u}
\end{array}\right]=-\left[\begin{array}{c}
F^{1} \\
\hline \vdots \\
\hline F^{I}
\end{array}\right],
$$

and $u_{k+1}=u_{k}+\delta u$, with blocks defined by

$$
J_{m, n}^{i, j}=\frac{\partial F^{i}\left(u_{k} ; \phi_{m}^{i}\right)}{\partial u^{j}}\left(\phi_{n}^{j}\right) \text { and } F_{m}^{i}=F^{i}\left(u_{k} ; \phi_{m}^{i}\right),
$$

for $n=1, \ldots, N^{j}, m=1, \ldots, N^{i}$ and $i, j=1, \ldots, I$. 


\subsection{Integration Domains}

Typically in finite element applications, the mixed variational forms, e.g., $a$ and $L$ in (7) and $F$ in (17), are given as sums of integrals over different subdomains $\Omega^{i}$. We assume that all variational forms can be represented by sums over mesh entities, for instance as sums of integrals over cells in a domain, see, e.g., [31]. For mixed dimensional problems, the subdomains $\Omega^{i}$ will have different topological dimensions $d^{i} \leqslant d$, where $d$ is the topological dimension of the parent domain $\Omega$. We introduce the notation $x=\left(x_{1}, \ldots, x_{d}\right)$ for the coordinates of a point $x \in \Omega$. In the following, the notation $\mathrm{d} x=\mathrm{d} x_{1} \times \cdots \times \mathrm{d} x_{d}$ is used in integrals over a $d$-dimensional domain. We use the notation $\mathrm{d} s$ to integrate over a co-dimension 1 subdomain of $\Omega$.

\subsection{From a Single Domain to a Mixed Dimensional Finite Element Pipeline}

The implementation of finite element discretizations of mixed domain problems within a high level framework such as, e.g., the FEniCS Project $[1,19,31]$ involves a number of new concepts and algorithmic extensions in comparison with single domain problems. We begin by giving an overview of these here. We also mention Figure 8 for an overview of the FEniCS user interface and pipeline for solving mixed domain and/or mixed dimensional problems.

Language abstractions. The UFL $[1,2]$ provides a flexible interface for finite element spaces and variational forms, facilitating the definition of weak forms through targeted abstractions (for representing, e.g., integration domains, function spaces, and form arguments). For instance, a UFL Functionspace is defined via a pairing of a reference finite element and a single domain. For coupling unknown fields defined over multiple domains, a mixed function space is required. We present a light-weight extension representing Cartesian products of function spaces as tuples of standard function spaces in Section 4.

Assembly. A standard finite element assembly process (see, e.g., [31]) relies on the insertion of local (element-wise) tensors, computed from a given weak form, into a global tensor via a local-toglobal degree-of-freedom mapping. The block structure of the systems (12) and (20) motivates a block-by-block assembly approach, and the decomposition of the variational form into subforms. Here, we present an automated block-by-block assembly algorithm, relying on dedicated extraction algorithms introduced in Section 4.3. The kernel for the computation of the local tensors for a given form depends on the integration domain type, and is detailed in Section 4.2. As for single domain problems, mixed domain local tensors of homogeneous dimension involve a single reference cell, and hence standard kernel implementations are applicable in that case. However, the cell assembly of codimension one mixed forms requires a revised approach. In short, local cell tensors over lower dimensional cells are here computed as local exterior facet tensors relative to the higher dimensional adjacent cells. This approach and the corresponding algorithms are detailed in Section 6.

Mappings for nested submeshes. The local-to-global degree-of-freedom mappings involved in the assembly algorithm maps the set of local basis function indices to the corresponding global indices. Off-diagonal blocks in (12) and (20) involve degrees of freedom from distinct domains, thus requiring the transfer of the associate local-to-global mappings between the corresponding submeshes. This is described in Section 5. The parent-child and sibling meshes mappings entailed in this transfer, together with the underlying data structures and algorithms, are introduced in Section 3.

\section{DATA STRUCTURES FOR NESTED SUBMESHES}

In this section, we discuss and suggest data structures for representing submeshes, in particular for nested submeshes. As detailed in Section 2.2, we consider the case where all subdomains $\Omega^{i}$ share 

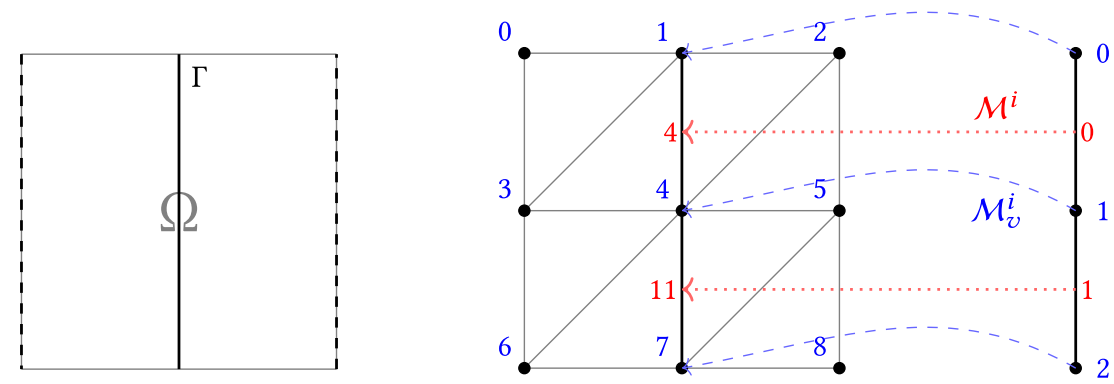

Fig. 2. Mapping between a submesh and its parent. The parent mesh $\mathcal{T}$ is the $2 \mathrm{D}$ mesh representing $\Omega$, while the submesh $\mathcal{T}^{1}$ represents the interface $\Gamma$ and can be constructed as a subset of $\mathcal{T}$ facets. The parent mesh has $n_{v}=9$ vertices $(\mathcal{V}=\{0, \ldots, 8\})$ and $n_{f}=16$ facets $(\mathcal{F}=\{0, \ldots, 15\})$, while the submesh $\mathcal{T}^{1}$ has $n_{v}^{1}=3$ vertices $\left(\mathcal{V}^{1}=\{0,1,2\}\right)$ and $n_{c}^{1}=2$ cells $\left(C^{1}=\{0,1\}\right)$. The mappings $\mathcal{M}_{v}^{1}: \mathcal{V}^{1} \rightarrow \mathcal{V}$ and $\mathcal{M}^{1}: C^{1} \rightarrow \mathcal{F}$ cf. (24) give $\mathcal{M}_{v}^{1}(0)=1, \mathcal{M}_{v}^{1}(1)=4, \mathcal{M}_{v}^{1}(2)=7$ and $\mathcal{M}^{1}(0)=4, \mathcal{M}^{1}(1)=11$.

a parent domain $\Omega$ and where each subdomain is covered by a conforming submesh $\mathcal{T}^{i}$ of the parent complex generated by $\mathcal{T}$. Two mappings between meshes are essential for mixed domain finite element assembly: first, mappings between submesh entities and parent mesh entities, and second, mappings between mesh entities in different submeshes. In the below, we formalize these concepts and describe their implementation in the FEniCS context.

\subsection{Mappings for Nested Submeshes}

We assume that each mesh is represented by the combination of its topology (defining the mesh entities and connections between these) and geometry (defining the spatial vertex coordinates) [30]. We denote by $n_{v}, n_{f}$, and $n_{c}$ the number of vertices, facets, and cells in $\mathcal{T}$, respectively, and let $\mathcal{V}$, $\mathcal{F}$, and $C$ be the corresponding sets of vertex, facet and cell indices:

$$
\mathcal{V}=\left\{v_{k}\right\}_{k=1}^{n_{v}}, \mathcal{F}=\left\{f_{k}\right\}_{k=1}^{n_{f}} \text { and } \mathcal{C}=\left\{c_{k}\right\}_{k=1}^{n_{c}} .
$$

We assume that any vertex, facet and cell can be identified by the respective index $v_{k}, f_{k}$, and $c_{k}$ and its (mesh entity) type. For each submesh, we denote by $\mathcal{V}^{i}$ and $C^{i}$ the sets of vertex and cell indices of $\mathcal{T}^{i}$, whose indices $v_{k}^{i}$ and $c_{k}^{i}$ are independent from the parent mesh numbering cf. (22):

$$
\mathcal{V}^{i}=\left\{v_{k}^{i}\right\}_{k=1}^{n_{v}^{i}}, C^{i}=\left\{c_{k}^{i}\right\}_{k=1}^{n_{c}^{i}} .
$$

We now introduce two maps that link the vertex indices in the submesh with the corresponding vertex index in the parent mesh, and the cell indices in the submesh with the corresponding mesh entity index in the parent mesh. In particular, for each submesh $\mathcal{T}^{i}$, we define its (child-to-parent) vertex map $\mathcal{M}_{v}^{i}$ and cell map $\mathcal{M}^{i}$ :

$$
\mathcal{M}_{v}^{i}: \mathcal{V}^{i} \rightarrow \mathcal{V} \text { and } \mathcal{M}^{i}: C^{i} \rightarrow C \text { or } \mathcal{F}
$$

We note that if the submesh $\mathcal{T}^{i}$ has the same topological dimension as its parent $\mathcal{T}$, each cell in the submesh is a cell in the parent mesh and $\mathcal{M}^{i}$ maps to $\mathcal{C}$. However, the cells of a submesh of codimension $e \geq 1$ are mesh entities of codimension $e$ in the parent mesh $\mathcal{T}$, and $\mathcal{M}^{i}$ maps to $\mathcal{F}$ when $e=1$. These concepts applied to the reference Example (1) are illustrated in Figure 2.

The map $M^{i}$ is injective, but not bijective (except in the trivial cases where the submesh is the entire mesh complex of a given dimension). We define the inverse maps $\left(M^{i}\right)^{-1}: C$ or $\mathcal{F}$ to $C^{i}$ via the natural restrictions (or empty extensions): for parent elements $e$ that have a representative $e^{i}$ in $\mathcal{T}^{i}$, we set $\left(M^{i}\right)^{-1}(e)=e^{i}$; for the rest, we set to it as undefined. Composite maps $\left(M^{i}\right)^{-1} \circ M^{j}$ for submeshes $T^{i}, T^{j}$ with $d^{i}=d^{j}=d$ should then be understood as maps from the cells in the 

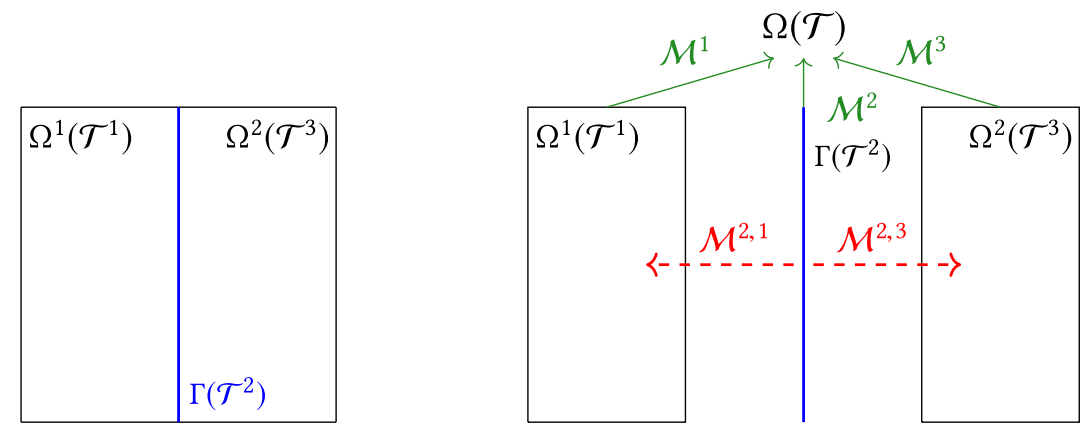

Fig. 3. The assembly of the systems can require additional mappings $\mathcal{M}^{i, j}$ to relate the submeshes $\mathcal{T}^{i}$ and $\mathcal{T}^{j}$ with $i \neq j$, assuming they are built from the same parent $\mathcal{T}$. For example, the additional mapping $\mathcal{M}^{2,1}$ : $C^{2} \rightarrow \mathcal{F}^{1}$ is needed and only built if $a^{1,2}\left(\phi_{n}^{2}, \phi_{m}^{1}\right)$ or $a^{2,1}\left(\phi_{m}^{1}, \phi_{n}^{2}\right)$ is non-zero, $n=1, \ldots, N^{2}, m=1, \ldots, N^{1}$.

submesh $T^{j}$ to the corresponding cell in the parent mesh and then, if included, to the corresponding cell in $T^{i}$. If there is no overlap between $T^{i}$ and $T^{j}$, this reduces to the empty map.

A mixed domain problem can couple an arbitrary but finite number $I$ of fields $u^{i} \in \Omega^{i}, i=$ $1, \ldots, I$. The assembly of the systems (12), (20) can then require additional mappings $\mathcal{M}^{i, j}$ to relate the submeshes $\mathcal{T}^{i}$ and $\mathcal{T}^{j}$ involved in, e.g., $a^{i, j}$ (8) for $i \neq j$ as illustrated by Figure 3, assuming their intersection $\mathcal{T}^{i} \cap \mathcal{T}^{j}$ is non-empty. Assume that $d^{j} \geq d^{i}$ without further loss of generality We can then express the map from cell indices of $\mathcal{T}^{i} \in \mathcal{T}^{i} \cap \mathcal{T}^{j}$ to corresponding mesh entity indices in $\mathcal{T}^{j}$ as

$$
\mathcal{M}^{i, j}: C^{i} \rightarrow C^{j} \text { or } \mathcal{F}^{j}
$$

As all submeshes $\mathcal{T}^{i}, i=1, \ldots, I$ share the same parent mesh $\mathcal{T}$ by assumption, we can use the mappings $\mathcal{M}^{i}$ and $\mathcal{M}^{j}(24)$ to establish the relation between $C^{i}$ and $C^{j}$. If $\mathcal{T}^{i}$ and $\mathcal{T}^{j}$ have the same topological dimension $\left(d^{i}=d^{j}\right)$, then the cell map between the two submeshes can be expressed directly as

$$
\mathcal{M}^{i, j}=\left(\mathcal{M}^{j}\right)^{-1} \circ \mathcal{M}^{i}: C^{i} \longrightarrow C^{j} .
$$

On the other hand, if $d^{i} \neq d^{j}$ i.e., $d^{i}=d^{j}-1$, then the computation of $\mathcal{M}^{i, j}$ requires additional intermediate steps. The computation of this mapping is performed as follows, and illustrated in Figure 4. The mapping $\mathcal{M}^{i}: C^{i} \rightarrow \mathcal{F}(24)$ gives the facet index $f_{k} \in \mathcal{T}$ (thus relative to the parent mesh) associated with the (lower dimensional) cell index $c_{k}^{i} \in \mathcal{T}^{i}$. The mesh connectivity, relating entities of various dimension within the same mesh, denoted as $(d-1) \rightarrow d$ in [30] from $\mathcal{F}$ to $C$, gives the indices of the $n_{s}$ cells adjacent to the facet $f_{k}$ in $\mathcal{T}$, with $n_{s}=1$ if $f_{k}$ is a boundary facet and $n_{s}=2$ otherwise. For higher dimensional gaps, $n_{s}$ can be arbitrary large (see Section 9.3). Next, for each of these cells $c_{k}^{i} \in C$, the inverse map $\left(\mathcal{M}^{j}\right)^{-1}: C \rightarrow C^{j}$ gives their corresponding indices $c_{k}^{j}$ in the submesh $\mathcal{T}^{j}$ (if present), $k \in\left[\left|1, n_{s}^{j} \leqslant n_{s}\right|\right]$. Note that $n_{s}^{j}$ is not necessarily equal to $n_{s}$, since $f_{k} \in \mathcal{T}$ can be a boundary facet in $\mathcal{T}^{j}$ while an interior facet in $\mathcal{T}$ (see Figure 4(d)). Finally, the facet $f_{k}^{j} \in \mathcal{F}^{j}$ shared by these cells can be found via the mesh connectivity $d^{j} \rightarrow\left(d^{j}-1\right)$ in $\mathcal{T}^{j}$.

\subsection{Nested Submesh Algorithms in FEniCS}

In this section, we discuss algorithms for nested submeshes and associated parent-child relationships in the context of the FEniCS/DOLFIN finite element library. The DOLFIN Mesh class provides data structures and algorithms for computational meshes holding the underlying geometry and topology through dedicated objects MeshGeometry and MeshTopology [30, 33]. 


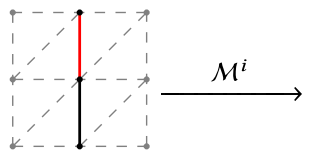

(a)

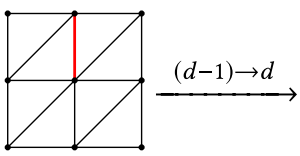

(b)

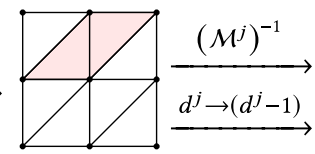

(c)

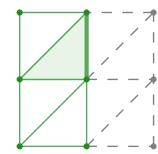

(d)

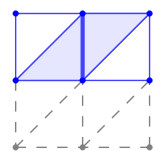

(e)

Fig. 4. Computation of the mapping $\mathcal{M}^{i, j}$ with $d^{i} \neq d^{j}$ i.e., $d^{i}=d^{j}-1$. The lower dimensional cell $c_{k}^{i} \in \mathcal{T}^{i}$ (a) is a facet $f_{k} \in \mathcal{T}$ (b) shared by two cells in the parent mesh $\mathcal{T}$ (c). The inverse mapping $\left(\mathcal{M}^{j}\right)^{-1}$ gives the equivalent of each of these cells in the submeshes (or an empty element) (d) and (e), and the mesh connectivity is used to obtain the corresponding facet, which can be either a boundary facet (d) or an interior facet (e).

To efficiently represent meshes for mixed domain discretizations, we introduce a new lightweight MeshView class. This class is designed to allow for representing and building submeshes $\mathcal{T}^{i}$ as new Mesh objects while storing their relationship with the parent mesh $\mathcal{T}$. A MeshView object links two meshes (for instance a submesh and its parent mesh, or two submeshes) by holding pointers to the parent (or associated) mesh $\mathcal{T}$ together with the vertex and cells maps $\mathcal{M}_{v}^{i}$ and $\mathcal{M}^{i}$. To reduce complexity, we consider one generation of meshes i.e., we only support parent-child and sibling meshes.

To construct a submesh, we assume that a MeshFunction defined over the parent mesh encodes the selected subset of mesh entities by an integer, referred to as a tag. The MeshView class implements a create function which builds the submesh $\mathcal{T}^{i}$ and its child-to-parent maps $\mathcal{M}_{v}^{i}$ and $\mathcal{M}^{i}$ (24) from this MeshFunction and the corresponding tag. This function returns a new Mesh object, with a pointer to the MeshView object in its MeshTopology, and its use is illustrated in Listing 1.

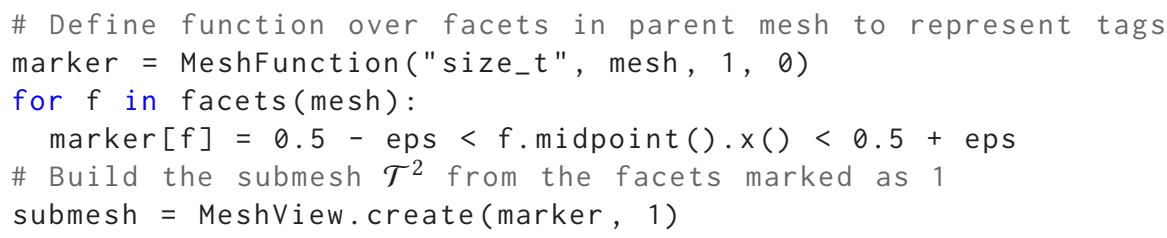

Listing 1. [Python] Creation of the submesh $\mathcal{T}^{2}$ from the parent mesh $\mathcal{T}$ (see Figures 2 and 3 ) for Example 1 using the MeshView class.

In addition to mapping between mesh entities of a submesh and its parent, mixed domain form assembly typically requires knowledge of mesh entity mappings between different submeshes as illustrated by Figure 3. We also represent these mappings via the MeshView class, and provide a new build_mapping function to construct these maps. In particular, for submeshes $\mathcal{T}^{i}$ and $\mathcal{T}^{j}$ with a shared parent mesh $\mathcal{T}$, build_mapping creates a new MeshView object pointing to the associated mesh $\mathcal{T}^{j}$, a cell map defined by (26) and an empty vertex map by default. This MeshView is then added to the map of MeshViews associated with the MeshTopology of submesh $\mathcal{T}^{i}$, in addition to its initial parent mesh view. Mappings between submeshes are built (and stored) on-the-fly during mixed domain form assembly. In particular, we do not build unnecessary mappings $\mathcal{M}^{i, j}: C^{i} \rightarrow$ $C^{j}$ or $\mathcal{F}^{j}$.

The algorithmic complexity of the submesh construction can be estimated as follows. The construction of each submesh $\mathcal{T}^{i}$ requires iterating over the $n_{c}^{i}$ marked entities in the parent $\mathcal{T}$. The mapping $\mathcal{M}^{i}(24)$ is then obtained and can be stored directly. The storage of the $n_{v}^{i}$ vertices of $\mathcal{T}^{i}$ to build the underlying MeshGeometry and the mapping $\mathcal{M}_{v}^{i}(24)$ requires iterating over the local 
vertices of each cell $c$ in the submesh $\mathcal{T}^{i}$, representing a complexity of $O\left(n_{v}^{i}\right)$. The MeshTopology holds the previously built mappings $\mathcal{M}^{i}$ and $\mathcal{M}_{v}^{i}$ and the numbering of $\mathcal{T}^{i}$ entities obtained directly by an iterative loop over both mappings whose complexity is $O\left(n_{v}^{i}+n_{c}^{i}\right)$.

\subsection{MPI-Parallelism of Nested Submeshes and Mappings}

DOLFIN [31] is designed to be seamlessly parallel, meaning that the same code can be used to perform both serial and parallel simulations. On distributed memory architectures, the parallel support relies on the Message Passing Interface (MPI). DOLFIN automatically performs mesh partitioning in parallel using the libraries ParMETIS [24] or SCOTCH [38]. Each processor holds only a portion of the global mesh, stored as a standard Mesh object, for which it is responsible. Data exchange between processors then requires the computation of local-to-global ${ }^{1}$ maps on each process. The nested submeshes $\mathcal{T}^{i}$ are assumed to be built from a common parent mesh $\mathcal{T}$. When running a mixed-dimensional simulation in parallel, the partitioning of the submeshes stems from the partitioning of the parent mesh $\mathcal{T}$, i.e., no auxiliary partitioning is performed. Thus, it is possible for a submesh to be distributed over only some of the available processors. And vice versa, it may be that a processor does not own any entities of a given submesh.

As described in Section 3.2, the submeshes $\mathcal{T}^{i}$ are represented as standard Mesh objects storing a MeshView in their MeshTopology. As for the global mesh $\mathcal{T}$, the submeshes $\mathcal{T}^{i}$ require a local-toglobal mapping to communicate data between processors, which implies establishing the ownership of each submesh entity among the processors. We assume that the cells can belong to only one partition, i.e., we do not introduce ghost cells. Each cell $c_{k}^{i}$ in $C^{i}$ is owned by the processor owning the corresponding entity $\mathcal{M}^{i}\left(c_{k}^{i}\right)$ in $\mathcal{T}$. However, the vertices located at the interface between partitions are shared by a set of processors. Among these, the vertices are assumed to be owned by the processor with the lowest rank. This processor holds the underlying local-to-global mapping and sends it to the other processors involved. Thus, a cell owned by a processor of rank $i$ may have vertices owned by a processor of $\operatorname{rank} j$, where $j<i$. This approach will lead to a slight load imbalance, but is not very significant, since the majority of vertices are not shared, and each processor only has a limited subset of mesh neighbors.

Since $\mathcal{M}^{i}$ and $\mathcal{M}_{v}^{i}$ are locally built on each processor, the global numbering within the scope of the parallel computation communicator needs additional loops and parallel communications to establish the ownership of the shared entities. Each additional mapping (26) requires iterating over the $n_{c}^{i}$ cells of $\mathcal{T}^{i}$ to find their counterparts in $\mathcal{T}^{j}, i \neq j$, hence representing a $O\left(n_{c}^{i}\right)$ complexity.

\section{FORM LANGUAGE ABSTRACTIONS AND ALGORITHMS FOR MIXED DOMAINS}

The UFL $[1,2]$ is a domain-specific language for finite element spaces, tensor algebra and variational forms. It provides a flexible interface for defining variational formulations of differential equations, through abstractions closely mimicking the mathematical syntax. UFL includes a set of predefined base finite element families, including but not limited to Lagrange [10], Discontinuous Galerkin [3], Raviart-Thomas [41], Brezzi-Douglas-Marini [11], and Nédelec [35, 36], of arbitrary polynomial order. The UFL finite element definition mimics that of Ciarlet [14], and in particular, a finite element is defined relative to a reference element (and not to a mesh). Mixed finite elements can be defined as Cartesian products of the base element families, assuming that all subelements share a common reference cell. A UFL function space is defined by a pairing of a (mixed) finite element and a domain (representing, e.g., the mesh). However, for mixed domain and dimensional problems, these abstractions are not sufficient.

To extend UFL with abstractions for mixed domain variational problems, we advocate a lightweight approach, essentially representing mixed function spaces (in contrast to function spaces

\footnotetext{
${ }^{1}$ In this section, local-to-global refers to process-to-communicator (local to process, global to communicator). ACM Transactions on Mathematical Software, Vol. 47, No. 4, Article 31. Publication date: September 2021.
} 
over mixed elements) as tuples of function spaces. This design choice mirrors our design choice for finite element assembly of mixed domain variational forms using block tensors. We detail the new UFL abstractions for mixed (domain) function spaces and integration in Sections 4.1 and 4.2 below. To facilitate mixed domain assembly, we have also extended UFL with new algorithms for splitting mixed domain variational forms into sums of subforms, described in Section 4.3. Finally, we describe UFL form validation and typical non-admissible operations in Section 4.4.

\subsection{Mixed Function Spaces and Functions}

To represent a mixed domain discrete function space $U=U^{1} \times \cdots \times U^{I}$ composed of a finite number $I$ of finite element function spaces $U^{i}$ for $i=1, \ldots, I$, we introduce a new ${ }^{2}$ UFL class MixedFunctionSpace. This lightweight class simply holds a tuple of the component spaces $\left(U^{1}, \ldots, U^{I}\right)$, and sample usage is provided in Listing 2 . The key operational aspect of the MixedFunctionspace abstraction is the identification of the relative position of a subfunction space and argument within the product space.

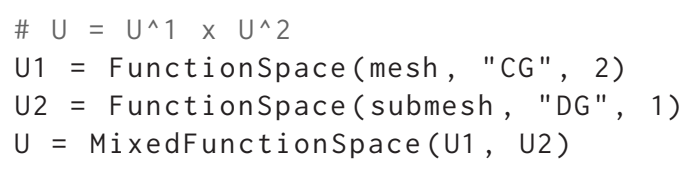

Listing 2. [Python] Example of MixedFunctionSpace usage in UFL/DOLFIN to define a mixed function space with two component spaces defined relative to different meshes.

UFL distinguishes between two types of functions appearing in variational forms: (i) Arguments representing the basis functions for a function space and (ii) Coefficients representing any function in a function space, i.e., a weighted linear combination of basis functions. A Trialfunction and TestFunction represent pre-indexed Arguments with index corresponding to the order of the argument in the form(s). UFL assumes that a variational form is always linear in its Arguments but possibly nonlinear in its Coefficients. To define test and trial functions on a mixed function space, and arguments in general, the syntax TestFunctions, TrialFunctions and Arguments have been adopted. This syntax is illustrated in Listing 3 below. These operators, when acting on a MixedFunctionspace with $I$ subspaces, return a tuple of basis functions $u^{i}$ for $i=1, \ldots, I$, as a list of Argument objects embedding the block index $i$ of the underlying function spaces $U^{i}$.

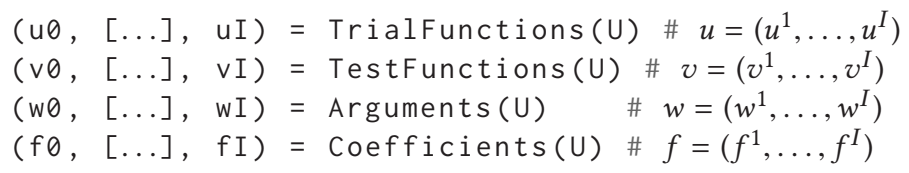

Listing 3. [Python]Basis functions (Arguments, and more specifically TestFunctions and TrialFunctions), or any functions (Coefficients) of a MixedFunctionSpace are defined as a list of functions embedding the block index of the underlying function space.

\subsection{Measures for Mixed Domain Variational Forms}

In UFL [2], integrals are expressed through multiplication $(*)$ by a measure representing the integral type. The main integral types are: $d x$ for integrals over the interior of the domain, $d s$ for the

${ }^{2}$ The keyword MixedFunctionSpace existed in previous versions of UFL, but was deprecated in version 2016.1.0. It has been reintroduced in a more generic context handling mixed domain and mixed dimensional function spaces. 
exterior facets i.e., the integrals over the boundary, and dS for the set of interior facets. Integrals over different parts of the domain can be expressed using markers given as an optional parameter subdomain_data and specifying the corresponding (integer) tag in the form expression. The terminal operands involved in the form integrals are Arguments and Coefficients, carrying their associated function space(s) and thereby the associated mesh(es) (see Listing 3). When the form arguments belong to the same function space i.e., for monodomain problems or for diagonal block forms, only one mesh is involved and the integration domain can thus be deduced without explicit definition by the measure.

For off-diagonal block forms, involving function spaces defined over different submeshes, the integration domain must be explicitly specified by measure (re)definition. Typical usage is illustrated in Listing 4.

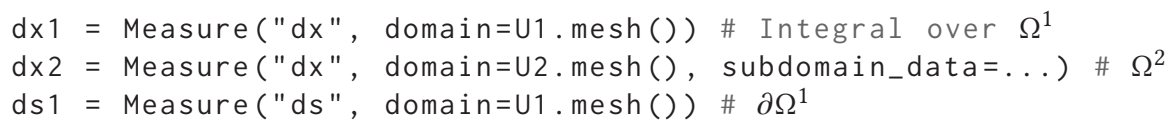

Listing 4. [Python] Example of redefining measures to define the integration domain of a form integral, giving explicitly the integral type, the integration mesh and possibly an integer marker to handle integrals over different parts of the domain. In this example, $d \times 1$ defines integrals over $\Omega^{1}, \mathrm{~d} \times 2(i)$ defines integrals over the part of $\Omega^{2}$ which has tag $i$ in the marker given to subdomain_data, and ds 1 defines integral over the boundary $\partial \Omega^{1}$.

When a measure is used in a form (integral), we assume that the measure's domain and the function space mesh of at least one of the form arguments coincide. Further, the redefined measures should define integration over cells for a lower dimensional mesh rather than integration over facets for a higher dimensional mesh. We note that all existing integral measures are supported for block forms involving a single mesh, but that interior facet integrals are not supported for off-diagonal block forms at the time of writing; more detail is given in the following, including in Remark 1 and Section 9.4.

With the measures defined in Listing 4, we can express the variational formulation of the Poisson problem introduced in Example 1 as follows (Listing 5).

$a=\operatorname{inner}(\operatorname{grad}(u), \operatorname{grad}(v)) * d \times 1+v * 1 * d \times 2(1)+u * e * d \times 2(1)$

Listing 5. [Python] UFL/DOLFIN implementation of the variational form (14) from Example 1 with explicit specification of the integration domains introduced in Listing 4.

\subsection{Mixed Domain Variational form Algorithms}

A key advantage of UFL and similar domain specific languages is the ability to manipulate, e.g., variational forms at the symbolic level. In the context of mixed domain variational forms, a key operation is to extract subform blocks i.e., to compute a decomposition into subforms (such as, e.g., (8)) of a mixed domain form. For instance, to assemble mixed domain variational forms, we advocate a block-by-block approach for the sake of flexibility, efficiency, and reuse. This is also the approach considered by $[5,27]$. For automated block-by-block assembly, the automated extraction of subforms from a variational form defined over a mixed function space is convenient.

To extract the subforms $a^{i, j}$ from a bilinear form $a$ (and the analogous for linear forms), we have introduced a UFL function extract_blocks. Its underlying algorithm relies on the directed acyclic graph (DAG) representation used by UFL $[2,31]$ to represent the form integrands, and in particular 
on the fact that that a form is linear in its arguments by definition. Our algorithm is also similar to that discussed in [22, Section 2]. For any given bilinear form $a$, its DAG expression tree allows the identification and extraction of the terms involving the pair $\left(U^{j}, U^{i}\right)$ of subspaces as the subform $a^{i, j}$, given the corresponding indexing $i, j=1, \ldots, I$. This algorithm relies on the embedding of the block index with the MixedFunctionSpace. The function extract_blocks can either return the whole list of subforms of a given form, or a specific subform $a^{i, j}$ given the indices $(i, j)$. A code example demonstrating the usage of this function is presented in Listing 6 below:

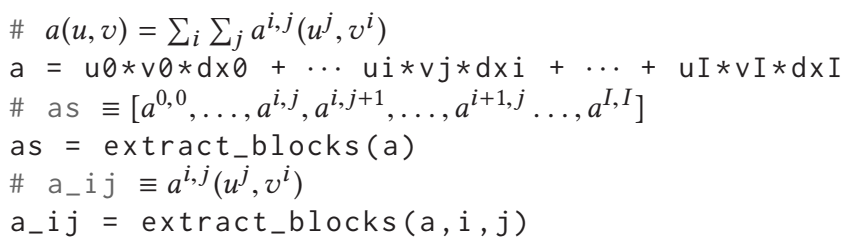

Listing 6. [Python] The extract_blocks function is used to extract the subforms $a^{i, j}$ of the mixed domains form $a(u, v)=\sum_{i} \sum_{j} a^{i, j}\left(u^{j}, v^{i}\right)$ from the arguments indexing. The subform $a^{1,2}$ from Example 1 implemented as in Listing 5 can be obtained using a_12 = extract_blocks $(a, 0,1)$. Note again that the indices start at 0 in the code.

\subsection{UFL Mixed Domain Form Verification}

The following code checks have been introduced to prevent confusion or misuse of the mixed domain features. All UFL verification assertions for single domain variational forms have been extended to mixed domain forms by application to each block subform.

Regarding the mixed function spaces definition, a MixedFunctionSpace is not a FunctionSpace, but rather a list of FunctionSpace objects. Arguments (resp. coefficients/functions) defined from a MixedFunctionSpace form a list of Arguments (resp. Coefficients) corresponding to each block. Thus, only the plural version of the related keywords are allowed: $\operatorname{TrialFunction}(V)$ is not allowed when $V$ is a MixedFunctionSpace. Instead, TrialFunctions $(V)$ should be used.

The coupling of arguments and/or functions from different function spaces in a form requires the underlying objects to be defined from a MixedFunctionspace. In other words, combining Trialfunction(V1) and TestFunction(V2) with V1 and V2 defined as different Functionspaces is not supported. One should instead introduce $V$ as a MixedFunctionSpace $\left(V_{1}, V_{2}\right)$ and define the arguments (TestFunctions $(V)$ and TrialFunctions $(V)$ ) from the latter.

The assembly of off-diagonal blocks combining arguments from different function spaces requires a mapping between the (sub)meshes involved, as discussed in Section 3. To define this mapping, we assume that the submeshes share a common parent mesh. This assertion is checked at the mesh data structure levels, e.g., when building mappings between the submeshes within the build_mapping function. Finally, a form integral is not valid if the integration mesh defined through the integral's measure does not coincide with one of the meshes associated with the form arguments.

\section{MIXED DOMAIN FUNCTION SPACES AND DEGREES OF FREEDOM}

In this section, we discuss the transfer of local-to-global degree-of-freedom maps between submeshes and introduce useful concepts and notation. These maps are used for the mixed domain assembly algorithms, described in detail using this notation in Section 6.

Consider a mesh $\mathcal{T}^{i}=\{K\}$ and a finite element space $U^{i}$ defined relative to $\mathcal{T}^{i}$. The standard local-to-global mapping $l_{c^{i}}^{i}$ for the finite element space $U^{i}$ and a cell $K \in \mathcal{T}^{i}$ with cell index $c^{i} \in C^{i}$ 


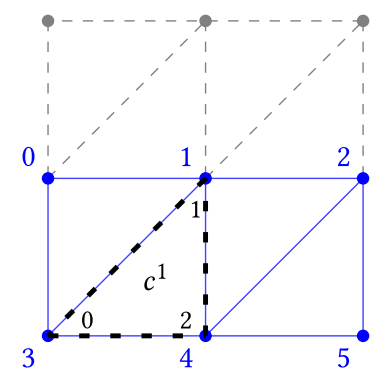

(a) $K$ in $\mathcal{T}^{1}$

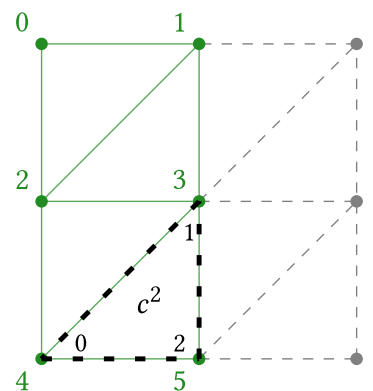

(b) $K$ in $\mathcal{T}^{2}$

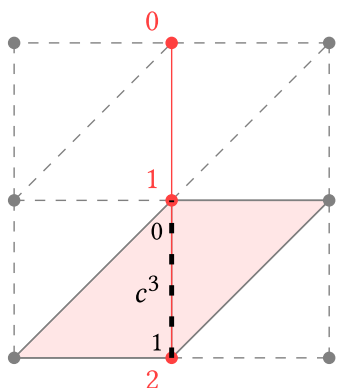

(c) $K$ in $\mathcal{T}^{3}$

Fig. 5. Illustration of submesh mappings and local-to-global degrees of freedom numbering. (a) The submesh $\mathcal{T}^{1}$ defined as the lower half of $\mathcal{T}$ with global degrees of freedom numbering (in blue) of $U^{1}\left(\mathcal{T}^{1}\right)$. The cell $K$ with index $c^{1} \in C^{1}$ has $N_{K}^{1}=3$ local degrees of freedom. The local-to-global relationship (30) gives $\iota_{c^{1}}^{i}(0)=3$, $\iota_{c^{1}}^{i}(1)=1$ and $\iota_{c^{1}}^{i}(2)=4$. (b) The submesh $\mathcal{T}^{2}$ defined as the left half of $\mathcal{T}$ with global degrees of freedom numbering (in green) of $U^{2}\left(\mathcal{T}^{2}\right)$. The cell $K$ with index $c^{2} \in C^{2}$ has $N_{K}^{2}=3$ local degrees of freedom (in black) with $\iota_{c^{2}}^{i}(0)=4, \iota_{c^{2}}^{i}(1)=3$ and $\iota_{c^{2}}^{i}(2)=5$. (c) The lower-dimensional submesh $\mathcal{T}^{3}$ defined as the middle vertical line of $\mathcal{T}$ with global degrees of freedom numbering (in red) of $U^{3}\left(\mathcal{T}^{3}\right)$. The cell $K$ with index $c^{3} \in C^{3}$ has $N_{K}^{3}=2$ local degrees of freedom (in black) with $\iota_{c^{3}}^{i}(0)=1$ and $\iota_{c^{3}}^{i}(1)=2$. The star $S_{K}$ of $K$ is composed of two cells (in light red).

maps the set of local basis function indices to the corresponding global indices:

$$
\iota_{c^{i}}^{i}:\left\{1, \ldots, N_{K}^{i}\right\} \rightarrow\left\{1, \ldots N^{i}\right\},
$$

where $N^{i}$ denotes the (global) dimension of $U^{i}$ and $N_{K}^{i}$ denotes the (local) dimension of $\left.U^{i}\right|_{K}$ for each $K$, see, e.g., [31] for more details. We assume that the local-to-global map $\iota_{c^{i}}^{i}$ is available for each submesh $\mathcal{T}^{i}$

First, consider the case of two submeshes $\mathcal{T}^{1}$ and $\mathcal{T}^{2}$ with a parent mesh $\mathcal{T}$ with $d=d^{1}=d^{2}$ as illustrated in Figure 5. Consider two function spaces $U^{1}=U^{1}\left(\mathcal{T}^{1}\right)$ and $U^{2}=U^{2}\left(\mathcal{T}^{2}\right)$. For each $K \in \mathcal{T}^{1}$ (resp. $K \in \mathcal{T}^{2}$ ) with index $c^{1} \in C^{1}$ (resp. $c^{2} \in C^{2}$ ), we can use the mapping $\mathcal{M}^{1}$ (resp. $\mathcal{M}^{2}$ ) to access the cell index $c$ relative to the parent mesh $\mathcal{T}$ :

$$
c=\mathcal{M}^{1}\left(c^{1}\right)=\mathcal{M}^{2}\left(c^{2}\right) .
$$

When there is a coupling between $\mathcal{T}^{1}$ and $\mathcal{T}^{2}$, the mapping $\mathcal{M}^{2,1}$ can be used to get the cell index $c^{1} \in C^{1}$ of $K$ relative to $\mathcal{T}^{1}$ from its index $c^{2} \in C^{2}$ relative to $\mathcal{T}^{2}$ :

$$
c^{1}=\mathcal{M}^{2,1}\left(c^{2}\right) \text {. }
$$

Subsequently, we can define the global index $n$ for the local (degree-of-freedom) index $l$ for $K$ in $U^{i}$ via

for $l=1, \ldots, N_{K}^{i}$.

$$
n=\iota_{c^{i}}^{i}(l)
$$

Next, consider the case of two submeshes $\mathcal{T}^{1}$ and $\mathcal{T}^{3}$ with a parent mesh $\mathcal{T}$ with $d=d^{1}>d^{3}$ and a function space $U^{1}$ defined relative to $\mathcal{T}^{1}$. For each $K \in \mathcal{T}^{3}$, we define its star $S_{K}$ as the set of cells in $\mathcal{T}$ containing $K$ (see Figure 5(c)). Take $\tilde{K} \in S_{K}$ and let $c \in C$ be its cell index relative to $\mathcal{T}$. By stipulation, $\tilde{K}$ is also a cell in $\mathcal{T}^{1}$, but its cell index $c^{1}$ relative to $\mathcal{T}^{1}$ is given by

$$
c^{1}=\left(\mathcal{M}^{1}\right)^{-1}(c) \text {. }
$$

This relation can thus be used to transfer local-to-global maps of degrees of freedom. 


\section{MIXED DOMAIN FINITE ELEMENT ASSEMBLY}

The finite element assembly of a variational form $a$ of arity $r$ is the computation of the $r$-tensor $A$ resulting from evaluating the variational form over its range of basis functions. A variational form can consist of multiple integrals, each with its own integration domain. A typical finite element assembly algorithm iterates over the cells $K$ of each integration domain $\mathcal{K}$ to compute the global finite element tensor $A$ by (i) evaluating local (cell-wise) element tensors $A^{K}$ and (ii) inserting (or adding) these into the global tensor via a local-to-global degree-of-freedom mapping. For more details on finite element assembly in general, see, e.g., [31].

\subsection{Mixed Domain Assembly Challenges and Discussion of Approach}

In single domain finite element assembly, the basis functions and coefficients are defined on one mesh $\mathcal{T}$ and the integration domains are defined relative to this mesh. For the assembly of mixed domain variational forms, we here consider a block-by-block approach as illustrated by, e.g., the decomposition (8) and the resulting block linear system (12) for bilinear forms and the general decomposition (9). In particular, we assemble each integral of each block form separately. Diagonal block forms are defined relative to a single domain, and can thus be assembled using standard single domain assembly algorithms. We therefore do not discuss these further here, but rather focus on the off-diagonal blocks. These present a number of additional challenges as follows:

- Assembly of off-diagonal blocks requires knowledge of the relationships between the integration mesh given by the form measure and the meshes involved in the trial and test spaces. These relationships are obtained through mappings between the parent-child or sibling meshes as discussed in Section 3.

- New techniques are required for the evaluation (and form compilation) of local element tensors over function spaces defined over different domains and dimensions.

In single domain finite element assembly, each local element tensor corresponds to the contribution from a single element. In mixed dimensional finite element assembly, the finite element tensor can again be formed by combining local element tensor contributions. However, the local element tensor concept is more multifaceted. Below, we introduce two local tensor concepts for mixed dimensional variational forms: the composite local element tensor and the local element tensor.

\subsection{Mixed Domain Assembly of Cell Integrals}

We analyze the assembly of a mixed domain variational block form of arity $r$ in further detail, using a bilinear form $(r=2)$ as a guiding case. The discussion is analogous for general $r$-forms. Consider an off-diagonal block form $a^{i, j}: U^{j} \times U^{i} \rightarrow \mathbb{R}$ for a fixed $i \neq j$ and assume without loss of generality that $\Omega^{i} \cap \Omega^{j} \neq \emptyset$, but that $\Omega^{i} \neq \Omega^{j}$. To alleviate notation, we just write $a=a^{i, j}$, and set $i=1$ and $j=2$, again without loss of generality. We further assume that $a$ represents a single integral, cf. Section 2.5 , noting that sums of integrals are easily handled. Thus, we have that

$$
a: U^{2} \times U^{1} \rightarrow \mathbb{R}, \quad a(\cdot, \cdot)=\sum_{K \in \mathcal{K}} a^{K}(\cdot, \cdot),
$$

where $\mathcal{K}$ is the integration domain of dimension $d^{\mathcal{K}}$, assumed to be (a subset of) the cells in either $\mathcal{T}^{1}$ or $\mathcal{T}^{2}$ (cf. Section 4.2). Specifically, we need to evaluate

$$
A_{m, n}^{K}=a^{K}\left(\phi_{n}^{2}, \phi_{m}^{1}\right)
$$

for all $m=1, \ldots, N^{1}, n=1, \ldots, N^{2}$. 


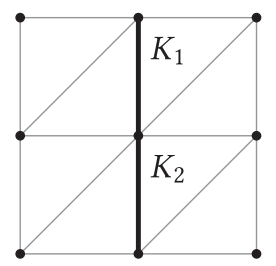

(a) $\mathcal{K}=\left\{K_{1}, K_{2}\right\}$ in $\mathcal{T}^{2}$

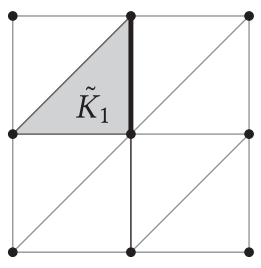

(b) $\tilde{K}_{1} \subset S_{K}$ in $\mathcal{T}^{1}$

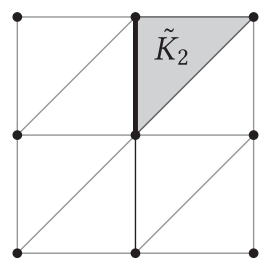

(c) $\tilde{K}_{2} \subset S_{K}$ in $\mathcal{T}^{1}$

Fig. 6. Mesh entities involved in the local element tensors of mixed dimensional terms for bilinear forms. To exemplify, we consider a parent mesh $\mathcal{T}$ of dimension $d=2$ and a continuous piecewise linear finite element space $U^{1}$ defined over a mesh $\mathcal{T}^{1}=\mathcal{T}$ of topological dimension $d^{1}=2$ and a continuous piecewise linear finite element space $U^{2}$ defined over a mesh $\mathcal{T}^{2}$ of topological dimension $d^{2}=1$. (a) Let $\mathcal{K}=\left\{K_{1}, K_{2}\right\} \subset \mathcal{T}^{2}$ be the integration domain. (b)-(c) For each $K \in \mathcal{K}$, we define its star $S_{K}=\left\{\tilde{K}_{1}, \tilde{K}_{2}\right\} \subset \mathcal{T}^{1}$ as the (two) adjoining cells in $\mathcal{T}^{1}$ each with $K$ as a facet.

6.2.1 Case 1 (Homogeneous Dimension) $d^{\mathcal{K}}=d^{1}=d^{2}$. If $\Omega^{1}$ and $\Omega^{2}$ both have the topological dimension of the integration mesh $d^{\mathcal{K}}=d^{1}=d^{2} \leq d$, the finite element spaces $U^{1}$ and $U^{2}$ can be defined over the same reference cell. In this case, the assembly of the local tensors $A^{K}$ (32) can be handled by standard techniques. Note that there is no implicit extension by zero: if $\Omega^{2} \subseteq \Omega^{1}$, fields defined over $\mathcal{T}^{2}$ cannot be integrated over cells in $\mathcal{T}^{1}$ that do not exist in $\mathcal{T}^{2}$ (i.e., not in $\Omega^{1} \cap \Omega^{2}$ ). The set $\mathcal{K}$ in (31) must then be (a subset of) the cells in $\mathcal{T}^{2}$. However, the insertion into the global tensor $A$ requires knowledge of the global degree of freedom numberings $n^{1}$ and $n^{2}$ relative to $U^{1}$ and $U^{2}$, respectively. These indices are obtained through the local-to-global mappings (30) as illustrated in Algorithm 1.

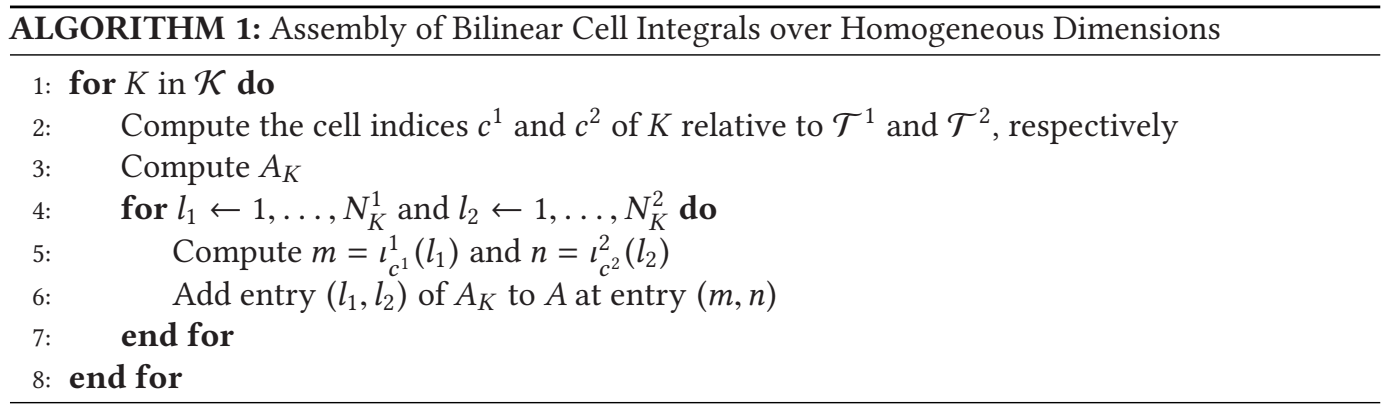

6.2.2 Case 2 (Codimension One). $d^{\mathcal{K}}=d^{2}=d^{1}-1$. Assume that $U^{1}$ and $U^{2}$ have different topological dimensions with $d^{1}>d^{2}$, and more specifically that $d^{1}=d^{2}+1$. The set $\mathcal{K}$ in (31) must then be (a subset of) the cells in $\mathcal{T}^{2}$ and (a subset of) the facets in $\mathcal{T}^{1}$. Each cell in $\mathcal{K}$ is either an interior facet in $\mathcal{T}^{1}$, in which case it is shared between two cells, or an exterior facet, in which case it belongs to a single cell and is located on the boundary of $\mathcal{T}^{1}$. We focus on the case of interior facets. The case of exterior facets is analogous but simpler. For instance, if $\mathcal{T}$ is a two-dimensional mesh of triangles, $\mathcal{T}^{1}$ is a (sub)mesh of triangles, and $\mathcal{T}^{2}$ is a (topologically onedimensional) submesh of intervals, then $\mathcal{K}$ must be a subset of the intervals in $\mathcal{T}^{2}$. This setting is illustrated for Example 1 in Figure 6.

Consider an element $K \in \mathcal{K}$. We note that $a^{K}\left(\phi_{n}^{2}, \phi_{m}^{1}\right)$ will be zero for all $m=1, \ldots, N^{1}, n=$ $1, \ldots, N^{2}$ for which $\left.\phi_{m}^{1}\right|_{K}=0$ or $\left.\phi_{n}^{2}\right|_{K}=0$. Conversely, $a^{K}\left(\phi_{n}^{2}, \phi_{m}^{1}\right)$ is potentially non-zero if $K$ is in the support of both $\phi_{m}^{1}$ and $\phi_{n}^{2}$ i.e., $K \subset \operatorname{supp}\left(\phi_{m}^{1}\right) \cap \operatorname{supp}\left(\phi_{n}^{2}\right)$. We denote the set of $U^{i}$ basis 
function indices with $K$ in their support by $\mathcal{N}_{K}^{i}$ i.e., $\mathcal{N}_{K}^{i}=\left\{n \in\left\{1, \ldots, N^{i}\right\} \mid K \subseteq \operatorname{supp}\left(\phi_{n}^{i}\right)\right\}$. Thus, potentially $a^{K}\left(\phi_{n}^{2}, \phi_{m}^{1}\right) \neq 0$ for $m \in \mathcal{N}_{K}^{1}$ and $n \in \mathcal{N}_{K}^{2}$, and $a^{K}\left(\phi_{n}^{2}, \phi_{m}^{1}\right)=0$ otherwise. Since $K \in \mathcal{T}^{2}$, the number of $U^{2}$ basis functions with $K$ in their support equals the local (cell) dimension of $U^{2}$ : $N_{K}^{2}=\operatorname{dim}\left(\mathcal{N}_{K}^{2}\right)=\operatorname{dim}\left(\left.U^{2}\right|_{K}\right)$. On the other hand, for $K$ viewed as an interior facet in $\mathcal{T}^{1}$, we define its star $S_{K}=\left\{\tilde{K}_{1}, \tilde{K}_{2}\right\}$ as the set of the (two) cells in $\mathcal{T}^{1}$ with $K$ as a facet (see Figure 6). The number of $U^{1}$ basis functions with $K$ in their support equals the dimension of $U^{1}$ restricted to the star: $N_{S_{K}}^{1}=\operatorname{dim}\left(\mathcal{N}_{K}^{1}\right)=\operatorname{dim}\left(\left.U^{1}\right|_{S_{K}}\right)$. For exterior facets, we simply define the star as the single cell with $K$ as a facet. To proceed, we introduce two new local tensor concepts for mixed dimensional variational forms. We define the composite local element tensor $A_{\varkappa, l}^{K}$ as the (potentially) non-zero contributions from the cell $K \in \mathcal{K} \subseteq \mathcal{T}^{2}$ to the global tensor:

$$
A_{\varkappa, l}^{K}=a^{K}\left(\phi_{n}^{2}, \phi_{m}^{1}\right), \quad m=\iota_{\tilde{c}}^{1}(\varkappa), n=\iota_{c^{2}}^{2}(l), \quad \varkappa=1, \ldots, N_{S_{K}}^{1}, l=1, \ldots, N_{K}^{2},
$$

where $\iota_{\tilde{c}}^{i}$ is a map from (local) degree-of-freedom indices of cells $\tilde{K} \subset S_{K}$ with index $\tilde{c}$ to global degree-of-freedom indices for $U^{i}$ (here for $i=1$ ). Subsidiary, for each $K \in \mathcal{K}$ and for each $\tilde{K} \in S_{K}$, we define the local element tensor $A_{k, l}^{\tilde{K}, K}$ as

$$
A_{k, l}^{\tilde{K}, K}=a^{K}\left(\phi_{n}^{2}, \phi_{m}^{1}\right), \quad m=\iota_{c^{1}}^{1}(k), n=\iota_{c^{2}}^{2}(l), \quad k=1, \ldots, N_{\tilde{K}}^{1}, l=1, \ldots, N_{K}^{2},
$$

for any $\tilde{K} \in \mathcal{T}^{1}$ with index $c^{1}$ and $K \in \mathcal{K} \subseteq \mathcal{T}^{2}$ with index $c^{2}$. We note that $m=\iota_{c^{1}}^{1}(k)$ can be computed via the submesh mapping transfer of the local-to-global mapping as described in Section 5. Since $K \in \mathcal{T}^{2}$, the local-to-global mapping $\iota_{c^{2}}^{2}$ is immediately available. The composite local element tensor can be expressed in terms of the local element tensors as

$$
A_{\varkappa, l}^{K}=A_{k, l}^{\tilde{K}, K}, \quad \varkappa=\gamma^{1}(k), k=1, \ldots, N_{\tilde{K}}^{1}, l=1, \ldots, N_{K}^{2}, \quad \tilde{K} \in S_{K},
$$

where $\varkappa=\gamma^{1}(k)$ is an appropriate map of local basis function indices on $\tilde{K}$ to composite local basis function indices on $S_{K}$. However, we note that the composite local element tensor $A^{K}$ need not be formed explicitly; rather selected parts of the local element tensors $A^{K, \tilde{K}}$ for $\tilde{K} \in S_{K}$ can be added directly to the global tensor.

To avoid counting the same contribution twice when directly adding the local element tensors $A^{\tilde{K}, K}$ to the global tensor $A$, the following approach may be used. If the star is composed of two cells $S_{K}=\left\{\tilde{K}_{1}, \tilde{K}_{2}\right\}$ as shown in Figure 6, the local tensor $A^{\tilde{K}_{2}, K}$ contains entries that have already been added to the global tensor when adding $A^{\tilde{K}_{1}, K}$. In particular, the entries $A_{k_{2}, l}^{\tilde{K}_{2}, K}, l=1, \ldots, N_{K}^{2}$ must be replaced by zero when there exists a $k_{1} \in\left\{1, \ldots N_{\tilde{K}_{1}}^{1}\right\}$ such that $\gamma^{1}\left(k_{2}\right)=\gamma^{1}\left(k_{1}\right)$ (see Figure 7).

Example 2. To illustrate the above ideas and concepts, we consider the computation of the finite element tensors for Example 1. We let $U^{1}$ and $U^{2}$ be finite element spaces of continuous piecewise linears defined relative to $\mathcal{T}^{1}$ and $\mathcal{T}^{2}$, respectively, and consider the off-diagonal matrix block $A^{1,2}$ corresponding to the form $a^{1,2}$ for each cell $K \in \mathcal{T}^{2}$ (corresponding to interior facets in $\mathcal{T}^{1}$ ):

$$
a^{K, 1,2}\left(\phi_{n}^{2}, \phi_{m}^{1}\right)=\int_{K} \phi_{n}^{2} \phi_{m}^{1} \mathrm{~d} x, \quad \forall m \in N^{1}, n \in N^{2} .
$$

Again, for readability, we drop the superscript ${ }^{1,2}$ in the following. The composite local element tensors of $a$ can be computed by selecting contributions from the elements of the star $S_{K}$, as illustrated in in Figure 6 and Figure 7. For this specific set of $U^{1}$ and $U^{2}, \operatorname{dim}\left(\mathcal{N}_{K}^{1}\right)=4$ and $\operatorname{dim}\left(\mathcal{N}_{K}^{2}\right)=2$.

In conclusion, a standard cell-wise finite element assembly algorithm can be augmented by an additional inner loop over adjacent mesh entities (stars) to allow for assembly of mixed dimensional cell integrals. The algorithm is given in Algorithm 2. 


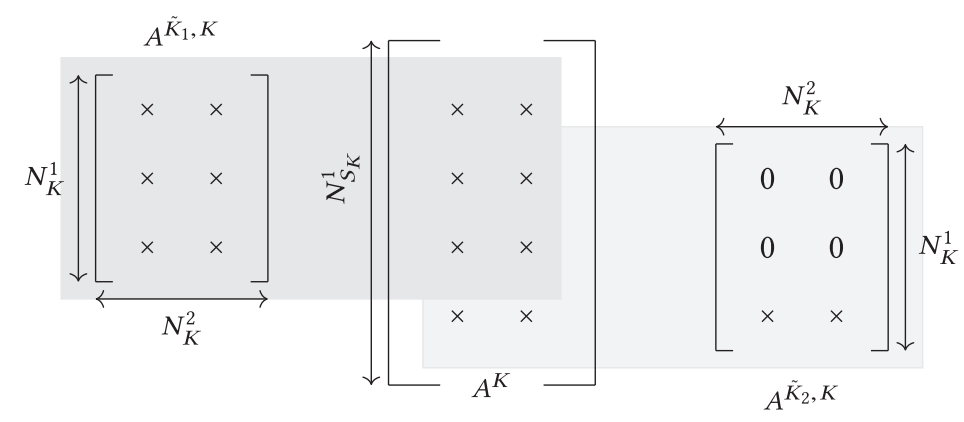

Fig. 7. Non-zero contributions to the element tensors for mixed dimensional (bilinear) forms cf. Example 1 and Figure 6. The composite local element tensor $A^{K}$, corresponding to the potentially non-zero entries of $A^{K}$ for $K \in \mathcal{K} \subseteq \mathcal{T}^{2}$ can be defined by selecting contributions from $\tilde{K}_{1}$ and $\tilde{K}_{2}$. $N_{K}^{i}$ is the local (elementwise) dimension of $U^{i}$ for $i=1,2$. The entries of the local tensor $A^{\tilde{K}_{2}, K}$ that have already been accounted for though $A^{\tilde{K}_{1}, K}$ can be zeroed in order to avoid adding a contribution twice.

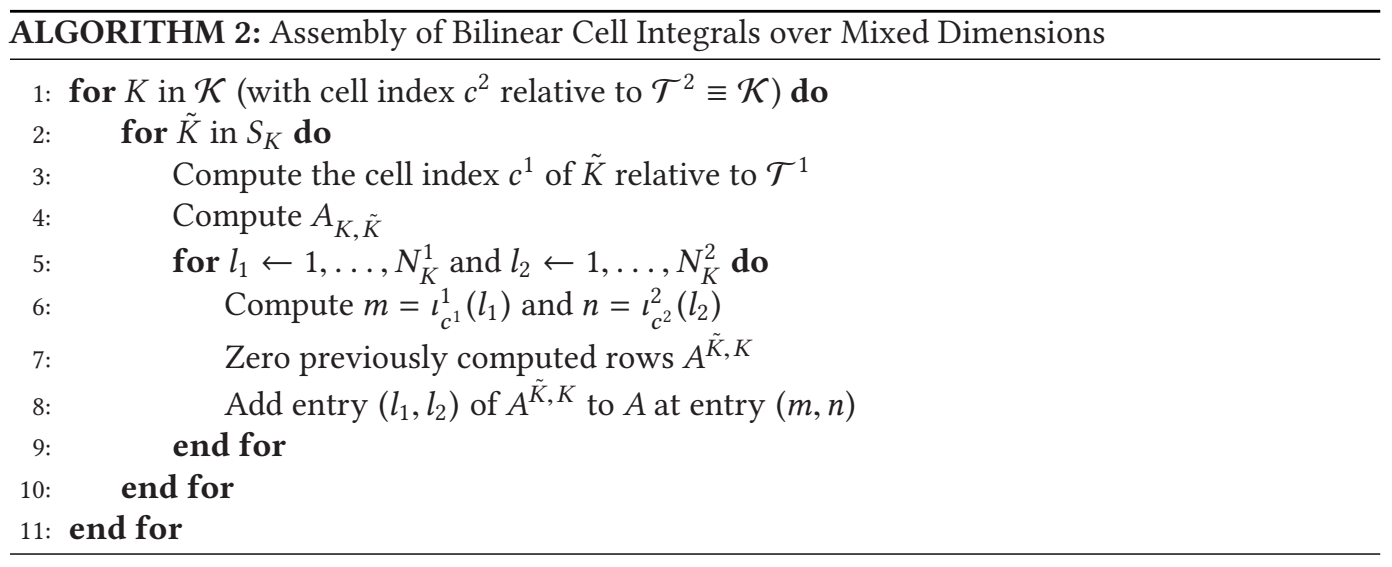

The case where $U^{1}$ and $U^{2}$ have the same topological dimensions $d^{1}=d^{2}$, but the integration domain is of lower dimension $d^{\mathcal{K}}<d^{1}=d^{2}$ can be viewed as an extension of the previous Case 2 where the star $S_{K}=\left\{\tilde{K}_{1}, \tilde{K}_{2}\right\}$ is handled as $S_{K}=S_{K}^{1} \cup S_{K}^{2}$ where $S_{K}^{1}=\left\{\tilde{K}_{1}\right\}$ (resp. $\left.S_{K}^{2}=\left\{\tilde{K}_{2}\right\}\right)$ is the star relative to $\mathcal{T}^{1}$ (resp. $\mathcal{T}^{2}$ ). Nevertheless, the trace on the integration domain is possibly not single-valued. Similarly, the case of codimension one i.e., $d^{1}=d^{2}+1$ with $U^{1}$ discontinuous, e.g., of type DG or $H_{\text {curl }}$ induces the trace on $U^{2}$ to not be single-valued. In the monodomain pipeline, the interior facet assembly involving discontinuous function spaces imposes the user to explicitly specify restrictions $[22,37]$ that are not supported with the cell assembly approach presented in this article. This limitation is further discussed in the dedicated Section 9.4.

Remark 1 (Assembly of Facet and Vertex Integrals). The dedicated algorithms presented here for mixed dimensional assembly focus on cell integrals, assuming other integral types to be defined as cell integrals over a lower-dimensional mesh. Standard assembly algorithms may handle various other integral types, e.g. facet or point assembly. We remark that these other types of integrals can be used as usual for diagonal blocks as the latter rely only on single domain assembly algorithms.

Remark 2 (Mixed Dimensional Assembly with Higher Dimensional Gaps). In general, and in particular for submeshes with codimension more than one, the star $S_{K}$ may contain an arbitrary 
number of cells in $\mathcal{T}$ sharing the lower dimensional cell $K$. We emphasize that the algorithms we have presented to form the composite local tensors $A^{K}$ can be applied to stars $S_{K}$ with an arbitrary number of elements. In particular, this design easily allows for extensions to higher dimensional gaps, e.g., assembly of coupled 3D-1D variational forms.

\section{OVERVIEW OF NEW FEniCS USER INTERFACE AND PIPELINE}

The abstractions and algorithms presented in this article have been implemented in the FEniCS finite element framework [1]. Both low-level and high-level features are available in $\mathrm{C}++$ and Python. Use of the high-level interface for solving mixed dimensional variational problems is exemplified in Listing 7, which solves the reference Poisson problem introduced in Section 1 (Example 1).

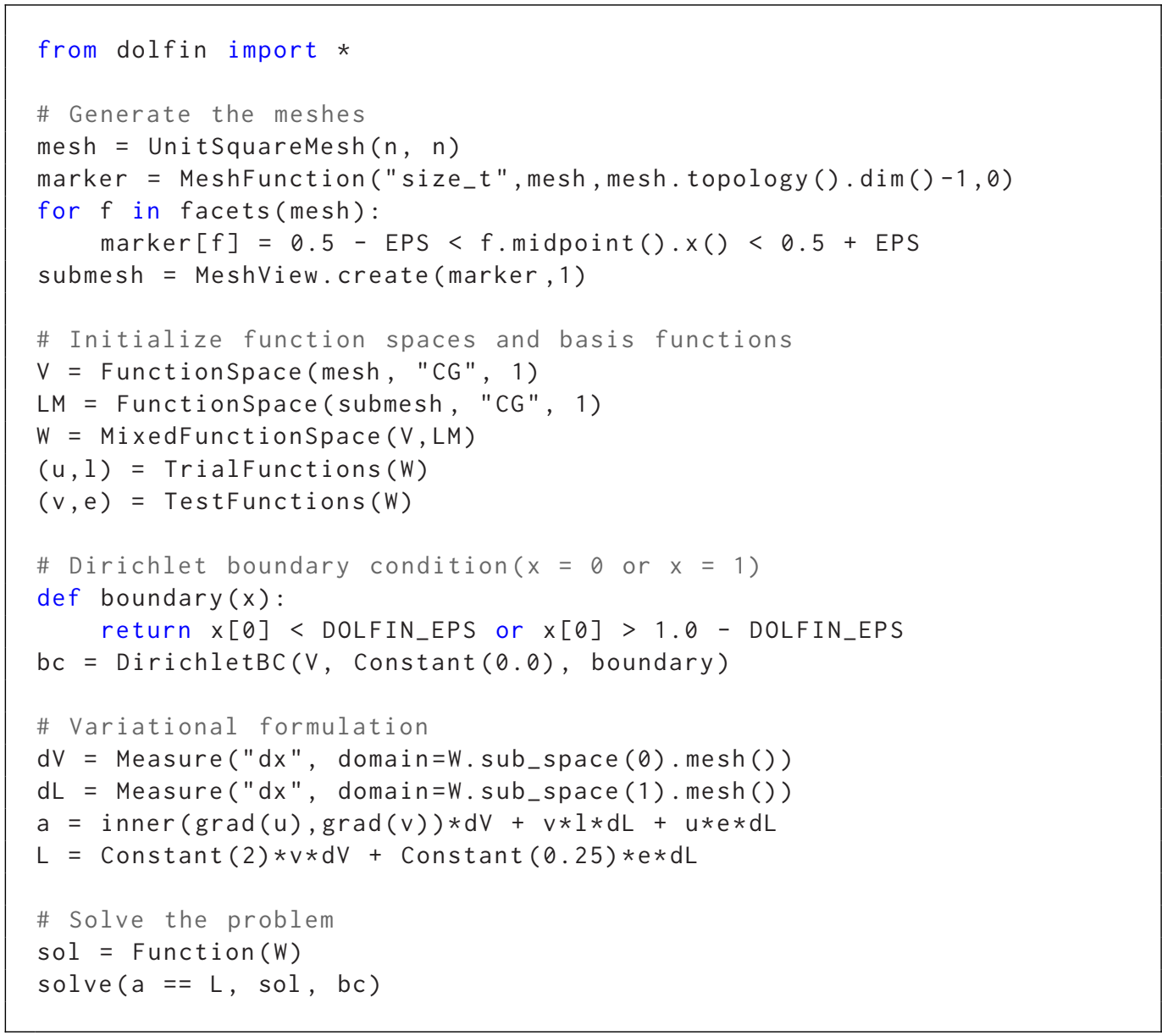

Listing 7. [Python] Illustration of mixed dimensional functionalities usage to solve the reference Poisson problem introduced as Example 1. The dedicated abstractions easily integrate the language, keeping the code syntax close to the mathematical formulation.

The solve function in the last line of Listing 7 encapsulates the whole mixed domains problem solving process including the extraction of block forms (see Section 4), the compilation and code generation for each resulting subform, the block-by-block assembly (see Section 6), and the solving of the recombined block system using a given solver. The presented framework covers a wide 
range of applications, and for the sake of flexibility, intermediate lower-level functions are also available. For instance, access to and manipulation of the separate matrix blocks can be used for preconditioning and iterative solution purposes. The overall structure of the mixed domain functionality is illustrated in Figure 8, while more implementation details are presented in the next sections.

\subsection{Code Generation of Local Tensors for Mixed Dimensional Forms}

The code for computing of the local tensors $A^{K}$ (tabulate_tensor) together with related quantities required for the assembly is auto-generated by the form compiler FFC [32] given a variational form. Hence, each variational form has its own kernel implementing the computation of the corresponding local tensors $A^{K}$ depending on the finite element, the integration domain and the form itself. In particular, the measures $\mathrm{dx}, \mathrm{ds}$, and dS discussed in Section 4.2 to represent the different integral types (cell integral, integral over exterior facets, and over interior facets, respectively) are mapped to different implementations of the tabulate_tensor function with appropriate signatures.

The algorithms presented in this article focus on cell assembly, in line with the stipulation that measures in mixed-dimensional forms define integration over cells of the lower dimensional mesh. The cell assembly of mixed forms with homogeneous dimension involves a single reference cell whose local tensors $A^{K}$ are computed as usual using the cell integral's tabulate_tensor function [32], with the local tensor A to be computed, information about the cell geometry, the coordinates of its degrees of freedom and the form coefficients, if any, as arguments. On the other hand, the codimension one local tensors $A^{\tilde{K}, K}$, for the cells $\tilde{K}$ in the star $S_{K}$, can be assembled as exterior facet integrals over the lower dimensional cell $K$. To accommodate for such computations, the tabulate_tensor signature for cell integrals has been revised, see Listing 8. In particular, we have added an optional input argument local_facet corresponding to the local facet index to mimic the tabulate_tensor implementation for exterior facet integrals.

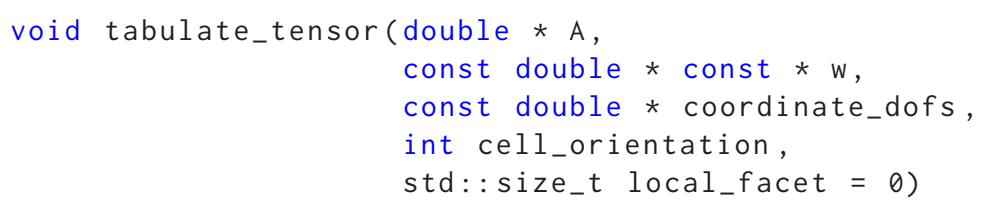

Listing 8. $[\mathrm{C}++]$ Signature of the revised tabulate_tensor function dedicated to local tensor computation of cell integrals for handling codimension one local tensors.

\subsection{FEniCS Interface to Mixed Domains Assembly}

The FEniCS assembler implementation has been revised to tackle mixed domain assembly of cell integrals as described in Section 6. We present the revised assembly algorithms for the case of homogeneous dimension (but mixed domains) and heterogeneous dimension (mixed dimensional) in the respective sections below.

The insertion of the local tensors $A^{K}$ and $A^{\tilde{K}, K}$ into the block tensor requires the local-to-global degree of freedom mappings discussed in Section 5. The cell indices $c^{i}$ relative to $\mathcal{T}^{i}$ required for the mappings $i_{c^{i}}^{i}(27)$ are stored in a double-indexed array cell_index[i][j]. The first index i represents the submesh $\mathcal{T}^{i}, i=0$ (resp. $i=1$ ) corresponding to the test (resp. trial) function space. The second index $j$ denotes the $j$ th contribution $\tilde{K}$ of $S_{K}$ in the case of heterogeneous dimension. These cell indices are obtained from the parent-child and sibling mesh mappings introduced in Section 3. 


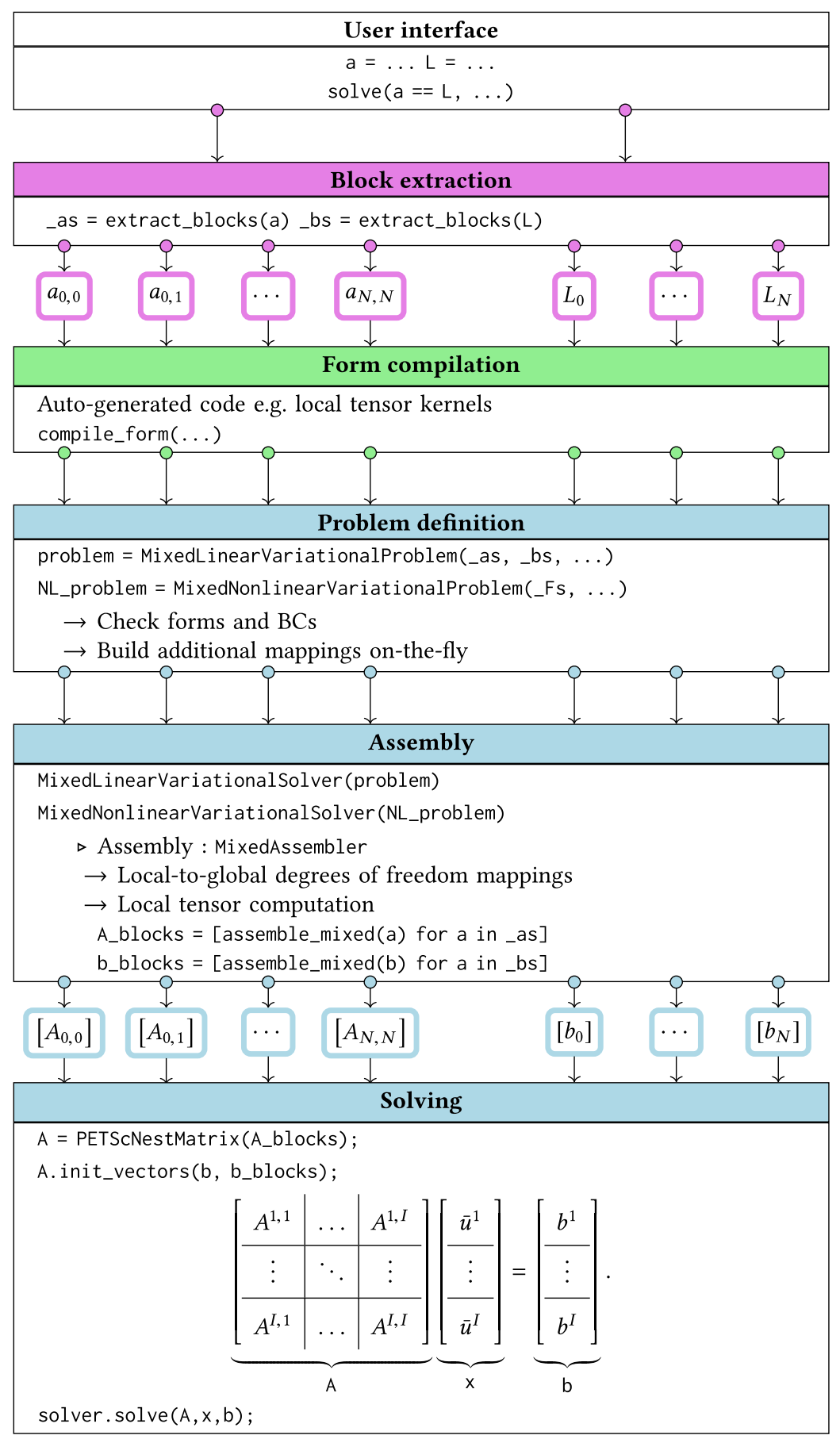

Fig. 8. Overview of the FEniCS user interface for mixed dimensional problems. The block colors refer to the FEniCS component impacted : ::UFL •:FFC •:DOLFIN. 
7.2.1 Case 1 (Homogeneous Dimension) $d^{\mathcal{K}}=d^{1}=d^{2}$. Only one cell $K$ is involved in the computation of each local tensor when the finite element spaces $U^{1}$ and $U^{2}$ are defined over the same reference element. The indices $c^{i}$ are the indices of this integration cell $K$ in the corresponding submeshes $\mathcal{T}^{i}$ (see Figure 5). The computation of these indices from the mesh mappings is shown in Listing 9.

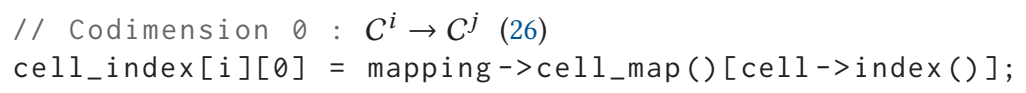

Listing 9. $[\mathrm{C}++]$ Each index $c^{i}$ (cell_index[i][0]) of the integration mesh cell $K$ (cell) relative to the basis function space mesh $\mathcal{T}^{i}$ is computed from the cell maps provided by the corresponding MeshView object mapping.

As described in Section 6, the cell assembly algorithm iterates over the cells $K$ of the integration domain $\mathcal{K}$ (mesh). The computation of the local tensor $A^{K}$ relative to the integration mesh cell $K$ use the standard implementation of tabulate_tensor kernel. The indices $m$ and $n$ (e.g., in the case of bilinear forms) of the global degrees of freedom in the function spaces $U^{i}$ for $i=1,2(i=0,1)$ are obtained from the local-to-global mappings $\iota^{i}(27)$.

7.2.2 Case 2(Codimension One) $d^{\mathcal{K}}=d^{2}=d^{1}-1$. The codimension one cell assembly involves the star $S_{K}$ of the integration mesh cell $K$ (see Figure 5). The computation of the indices (cell_index [i][j]) of the cells $K^{j}$ in the star $S_{K}$ via the submesh mappings and the mesh connectivity is shown in Listing 10. The local index of $K$ viewed as a facet relative to $K^{j}$ (local_facets[j]), required by the revised tabulate_tensor implementation (see Listing 8), can also be derived from the mesh connectivity.

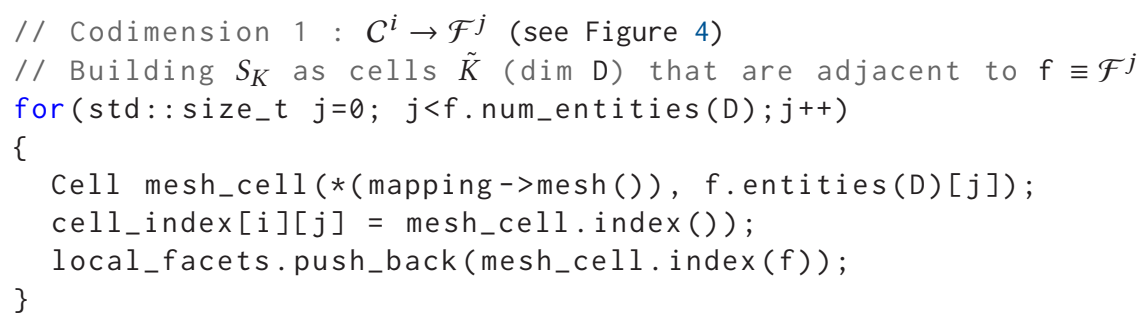

Listing 10. $[\mathrm{C}++]$ The index $c^{i}$ (cell_index[i][j]) of $K^{j} \in S_{K}$ relative to $\mathcal{T}^{i}$ is obtained from the set of cells adjacent to the corresponding facet $f \equiv \mathcal{F}^{j}$ in the higher dimensional mesh. The local index of this facet in the cell $K^{j}$ (local_facets $[j]$ ) is required to compute the local tensors $A^{K^{j}, K}$.

As detailed in Algorithm 2, the assembly over mixed dimensions involves an additional loop over the cells $\tilde{K}$ in $S_{K}$. The local tensors $A^{\tilde{K}, K}$ are computed from the revised tabulate_tensor function taking the local index of the corresponding facet as an additional argument (see Section 7.1). Again, the indices $m$ and $n$ (e.g., in the case of bilinear forms) of the global degrees of freedom in the function spaces $U^{i}$ for $i=1,2(i=0,1)$ are obtained from the local-to-global mappings $i^{i}(27)$. As specified in Section 6, entries of the local tensors $A^{\tilde{K}, K}$ may have to be zeroed to avoid duplicates. 


\subsection{Block Linear Algebra and Representing Assembled Tensors}

Once assembled as described in Section 6, the block tensors are recombined to form the block systems (12) and (20). The FEniCS/DOLFIN library [33, 34] uses the software package PETSc [4] for linear algebra objects and algorithms.

In particular, the PETSc MATNEST structure is dedicated to efficient representation and use of block tensors. This PETSc data structure has been interfaced in FEniCS/DOLFIN as a PETScNestMatrix class building a MATNEST object from a list of matrices, cf. Listing 11. The PETScNestMatrix class is also equipped with a init_vectors function for building vectors with the appropriate block structure.

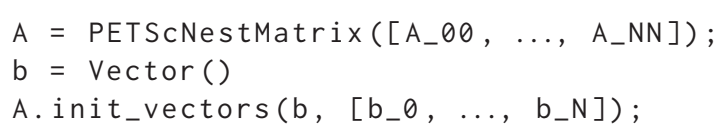

Listing 11. [Python] The assembled blocks are combined into a single block tensor of type PETScNestMatrix which interfaces the dedicated PETSc data structure MATNEST. The right hand side block vector $b$ is built from A using the init_vectors function with a matching structure.

The wide selection of solvers and preconditioners available in FEniCS/DOLFIN via PETSc can be applied to PETSCNestMatrix objects in the same manner as with standard single domain system. However, direct solvers are not directly compatible with this data structure. Instead, PETSCNestMatrix objects can easily be converted from the MATNEST format to the more standard MATAIJ format using the function convert_to_aij interfacing the corresponding PETSc routine (see Listing 12). When solving nonlinear problems, the function convert_to_aij is inherent to the use of a direct solver within the Newton solver iterations. A dedicated warning explicitly informs the user in that case.

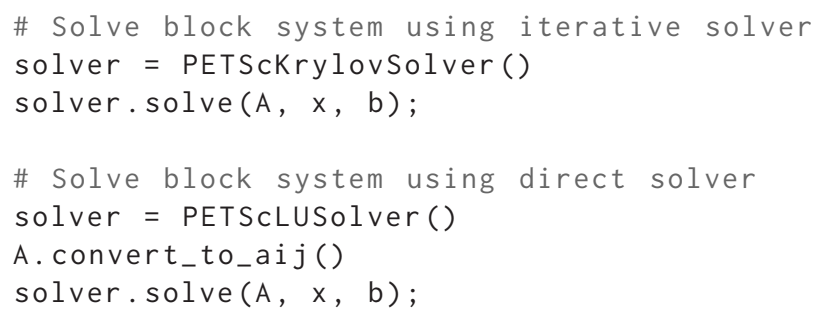

Listing 12. [Python] The recombined block system $A x=b$ can be solved using, e.g., iterative or direct solvers.

Moreover, the MATNEST data structure is compatible with the PCFieldSplit preconditioners offered by PETSc, allowing for the application of specific preconditioners to each block matrix.

\section{NUMERICAL RESULTS}

The presented framework is applicable to a wide range of mixed dimensional problems. In this section, we report on numerical results for three selected cases. First, we start with the reference Poisson problem introduced in Section 1 as a demonstration of the described features. Second, we study two Stokes problems with non-standard boundary conditions as discussed in [7, 47]. Finally, we consider a mathematical model of ionic electrodiffusion in brain tissue. Our software to reproduce the presented results is openly and freely available through the standard FEniCS distribution (development version) and the source code in [16]. 


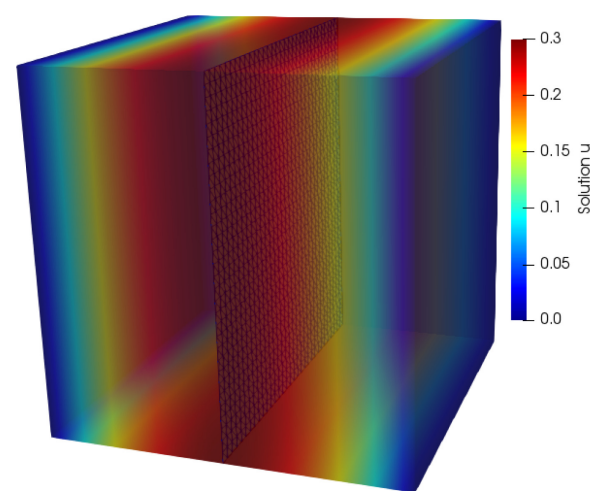

(a) Solution $u_{h}$

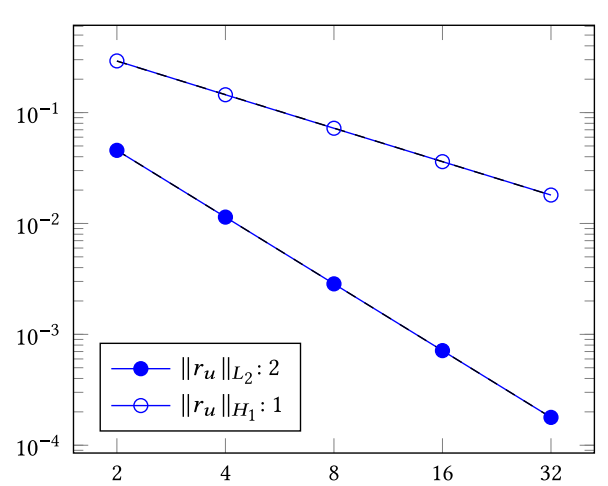

(b) Convergence study

Fig. 9. Solution of the Poisson problem given in Example 1 with $f=2$ and $c=0.25$ to obtain the manufactured solution $u(x, y)=x(1-x)$, and the corresponding convergence study plotting $\left\|e_{h}\right\|_{L_{2}}$ and $\left\|e_{h}\right\|_{H_{1}}$ with order $k=1$ depending on the mesh resolution. $\left\|r_{u}\right\|_{L_{2}}$ and $\left\|r_{u}\right\|_{H_{1}}$ are the resulting convergence rates.

\subsection{Reference Poisson Problem}

This numerical experiment presents a convergence study performed on the 3D version of Example 1 using the method of manufactured solutions. The function $u(x, y)=x(1-x)$ is the exact solution of Example 1 with $f=2$ and $c=0.25$. We consider a uniform tetrahedral mesh $\mathcal{T}^{1}$ of the unit cube, and find the approximations $u_{h}$ using the space of continuous piecewise linear polynomials $U^{1}$ relative to this mesh and $c_{h}$ using continuous piecewise linear polynomials defined relative to a mesh $\mathcal{T}^{2}$ of the two-dimensional midplane. The obtained solution is shown in Figure 9(a).

We introduce the approximation error $e_{h}$ defined as $e_{h}=u-u_{h}$. Given $k$ the polynomial order associated with $U^{1}\left(\mathcal{T}^{1}\right)$, and $h$ the mesh size, there exists a constant $\alpha>0$ such that

$$
\left\|e_{h}\right\|_{L_{2}} \leqslant \alpha h^{k+1} \text { and }\left\|e_{h}\right\|_{H_{1}} \leqslant \alpha h^{k} .
$$

Figure 9(b) plots the $L_{2}$ and $H_{1}$ norms of the approximation error with respect to the mesh resolution, for the case $k=1$. The slope of the corresponding lines-indicated in legend-shows that the expected orders of convergence (37) are obtained.

\subsection{Fluid Flow Problems with Non-Standard Boundary Conditions}

8.2.1 A Stokes-Brinkman Problem with a Manufactured Solution. We consider the StokesBrinkman problem (38) solved on the domain $\Omega=[0,1] \times[0,1]$.

$$
\begin{aligned}
-\Delta u+u-\nabla p & =f \text { on } \Omega, \\
\nabla \cdot u & =0 \text { on } \Omega .
\end{aligned}
$$

We introduce the manufactured solutions $u$ and $p$ (39) from which the right hand side $f$ in (38) and the boundary conditions (40) are defined as

$$
u=\left(\begin{array}{c}
\cos (\pi y) \sin (\pi x) \\
-\cos (\pi x) \sin (\pi y)
\end{array}\right), \quad p=\pi \cos (\pi x) \cos (\pi y) .
$$

We denote by $\Gamma_{t}$ and $\Gamma_{b}$ the top and bottom boundaries, respectively, on which we impose homogeneous Neumann conditions. Further, we set the velocity $g$ at the left (inlet) boundary $\Gamma_{\text {in }}$ to be the manufactured solution $\left.u\right|_{\Gamma_{\text {in }}}$, imposed through a Lagrange multiplier $\lambda \in \Gamma_{\text {in }}$. In addition, an 
inhomogeneous Neumann condition is imposed on the right (outlet) boundary $\Gamma_{\text {out }}$, with traction $h$ defined from (39):

$$
\nabla u \cdot n+p n=h \text { on } \Gamma_{\text {out }} .
$$

Combining these conditions with (38), we obtain a mixed dimensional problem with the variational formulation: find $(u, p, \lambda) \in U \times P \times L$ such that

$$
\begin{aligned}
\int_{\Omega} \nabla u \cdot \nabla v+u \cdot v & +p \nabla \cdot v+q \nabla \cdot u \mathrm{~d} x+\int_{\Gamma_{\text {in }}} \lambda \cdot v+\eta \cdot u \mathrm{~d} s \\
& =\int_{\Omega} f \cdot v \mathrm{~d} x+\int_{\Gamma_{\text {out }}} h \cdot v \mathrm{~d} s+\int_{\Gamma_{\text {in }}} \eta \cdot g \mathrm{~d} s,
\end{aligned}
$$

for all $(v, q, \eta) \in U \times P \times L$. We define a uniform mesh of the unit square $\mathcal{T}^{1}$, and a lowerdimensional mesh $\mathcal{T}^{2}$ of the boundary $\Gamma_{\text {in }}$. The velocity $u$ is approximated as $u_{h} \in U$ through continuous piecewise vector fields of polynomial order $k+1 \geq 2$ while continuous piecewise polynomials of order $k \geq 1$ are used for the pressure approximation $p_{h} \in P$. The Lagrange multiplier $\lambda$ is approximated in the space $L=L\left(\mathcal{T}^{2}\right)$ of continuous piecewise polynomials of order $k$ defined relative to $\mathcal{T}^{2}$.

Iterative solvers, such as, e.g., GMRES with ILU preconditioning, easily fail to converge for this problem due to the ill-conditioning of the system. A fractional preconditioning strategy was introduced in [28]. In Figure 10, we demonstrate that we obtain the expected order of convergence (37), both using a direct solver and using said preconditioning technique, for $k=1,2$.

8.2.2 A Stokes Problem with Non-Standard Traction Conditions. The next test case presents a variant of the Stokes problem (see Figure 11) discussed in [7, 47], involving non-standard traction boundary conditions at the inlet and outlet boundaries $\Gamma_{i n}$ and $\Gamma_{\text {out }}$, respectively. This precise formulation involving the symmetric velocity gradient may be useful in connection with, e.g., fluidstructure interaction problems [7].

The traction conditions are imposed using a Lagrange multiplier $\lambda_{\text {in }}$ (resp. $\lambda_{\text {out }}$ ) relative to the inlet $\Gamma_{\text {in }}$ (resp. the outlet $\Gamma_{\text {out }}$ ), acting on the tangential component of the velocity $u \cdot t \equiv u \times$ $n$. The standard no-slip condition $u=0$ is applied to the walls $\Gamma_{w}$, and a pressure difference is imposed between the inlet where $p=p_{\text {in }}$ and the outlet where we impose $p=p_{\text {out }}$. We also apply homogeneous Dirichlet boundary conditions for both Lagrange multipliers $\lambda_{i}$ and $\lambda_{\text {out }}$.

The variational formulation then reads: find $\left(u, p, \lambda_{\text {in }}, \lambda_{\text {out }}\right) \in U \times P \times L_{\text {in }} \times L_{\text {out }}$ such that

$$
\begin{aligned}
\mu \int_{\Omega}(\nabla u & \left.+\nabla u^{T}\right) \cdot\left(\nabla v+\nabla v^{T}\right)-p(\nabla \cdot v)-q(\nabla \cdot u) \mathrm{d} x \\
& +\int_{\Gamma_{\text {in }}} \lambda_{\text {in }}(v \times n)+\eta_{\text {in }}(u \times n) \mathrm{d} s+\int_{\Gamma_{\text {out }}} \lambda_{\text {out }}(v \times n)+\eta_{\text {out }}(u \times n) \mathrm{d} s \\
= & \int_{\Omega} f \cdot v-\int_{\Gamma_{\text {in }}} p_{\text {in }}(v \cdot n)-\int_{\Gamma_{\text {out }}} p_{\text {out }}(v \cdot n),
\end{aligned}
$$

for all $\left(v, q, \eta_{\text {in }}, \eta_{\text {out }}\right) \in U \times P \times L_{\text {in }} \times L_{\text {out }}$.

Again, we define a uniform mesh $\mathcal{T}^{1}$ of the domain $\Omega$ and lower-dimensional meshes $\mathcal{T}^{2}, \mathcal{T}^{3}$ for the boundaries $\Gamma_{\text {in }}, \Gamma_{\text {out }}$, respectively. The approximation of the velocity $u$ and the pressure $p$ of (42) uses the standard Taylor-Hood elements, i.e., second order (resp. first order) Lagrange finite elements for $u \in U$ (resp. for $p \in P$ ). The Lagrange multipliers are defined in continuous piecewise quadratic function spaces $L_{\mathrm{in}}=L_{\mathrm{in}}\left(\mathcal{T}^{2}\right)$ and $L_{\text {out }}=L_{\text {out }}\left(\mathcal{T}^{3}\right)$, ensuring the well posedness of the problem as detailed in [47]. 


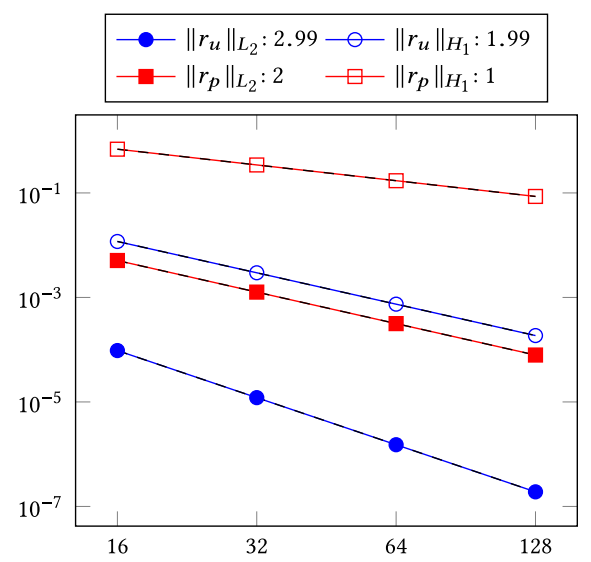

(a) $P_{2}^{2} \times P_{1} \times P_{1}^{2}$ - Direct solver

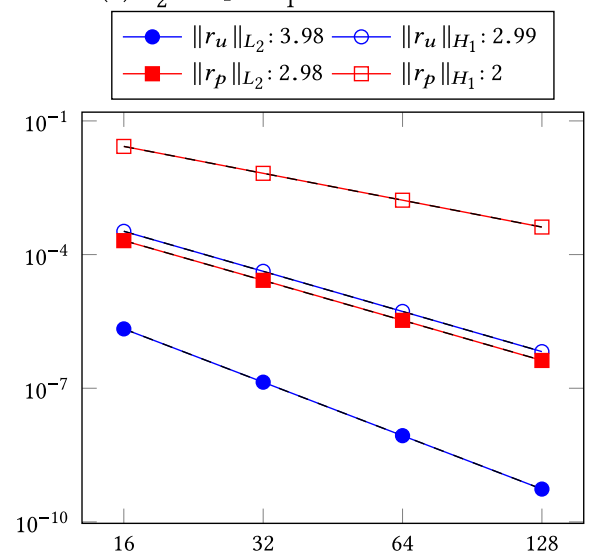

(c) $P_{3}^{2} \times P_{2} \times P_{2}^{2}$ - Direct solver

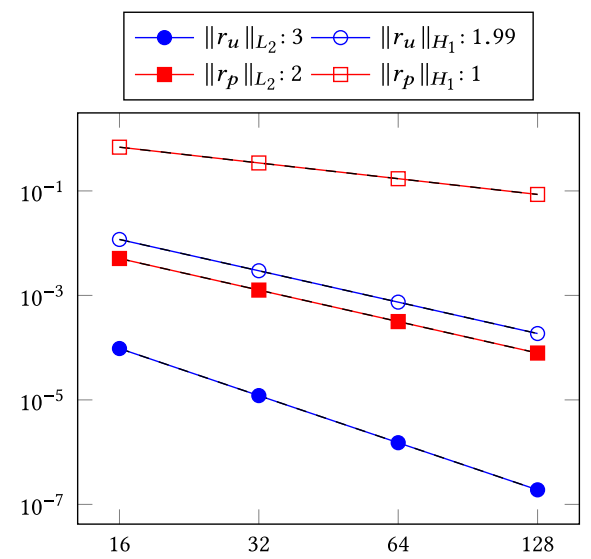

(b) $P_{2}^{2} \times P_{1} \times P_{1}^{2}$ - Preconditioned Minres

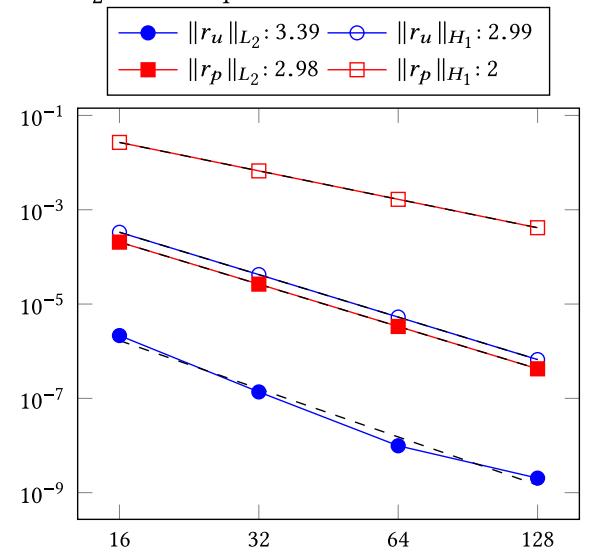

(d) $P_{3}^{2} \times P_{2} \times P_{2}^{2}$ - Preconditioned Minres

Fig. 10. Convergence study for the Stokes-Brinkman problem (38). The plots show the approximation error $\left\|e_{h}\right\|_{Q}$ (37) in the $Q$ norm depending on the mesh resolution. $\left\|r_{v}\right\|_{Q}$ defines the rate of convergence for the solution variable $v$ in the $Q$ norm. (a) and (b) show that the $P_{2}^{2} \times P_{1} \times P_{1}^{2}$ approximation gives the expected convergence rates in the $L_{2}$ and $H_{1}$ norms, both using a direct solver and a preconditioned [28] iterative solver. (c) and (d) show that the expected convergence rates are still obtained with higher order elements $P_{3}^{2} \times P_{2} \times P_{2}^{2}$.

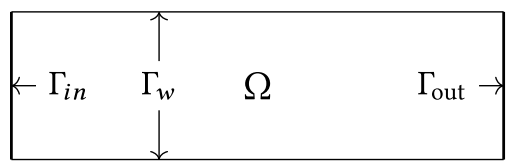

$$
\begin{aligned}
& -\mu \nabla\left(\nabla u+\nabla u^{T}\right)+\nabla p=f \text { on } \Omega \\
& \nabla \cdot u=0 \text { on } \Omega \\
& u \times n=0 \text { on } \Gamma_{\text {in }} \cup \Gamma_{\text {out }}
\end{aligned}
$$

Fig. 11. The domain $\Omega$ (left) is the rectangle $[0,10] \times[0.5]$ where $\Gamma_{\text {in }}$ and $\Gamma_{\text {out }}$ represent the inlet and the outlet, and $\Gamma_{w}$ denotes the upper and lower boundaries (representing horizontal walls). The equations (right) are based on a standard formulation of the Stokes equations with additional traction conditions on $\Gamma_{\text {in }}$ and $\Gamma_{\text {out }}$.

Figure 12 compares the results obtained with and without the traction condition, i.e., with and without the use of the Lagrange multipliers. Both solutions were computed through the presented framework and preconditioned with the fractional preconditioner [28] introduced in Section 8.2.1. 


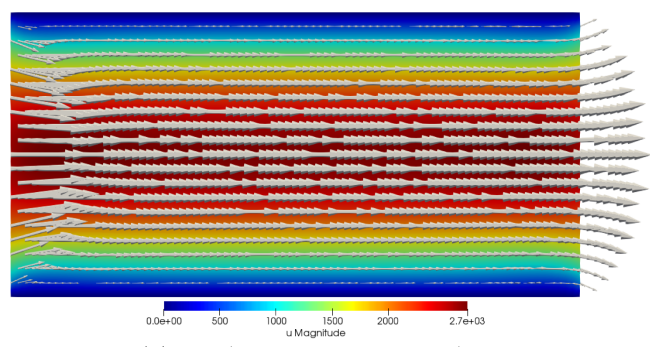

(a) Without traction condition

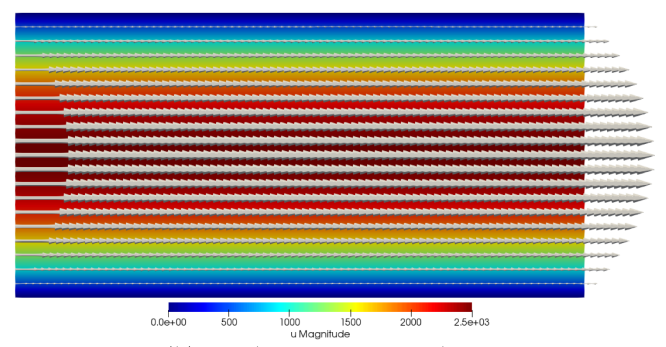

(b) With traction condition

Fig. 12. Comparison between the velocity field obtained from the Stokes problem with standard boundary conditions i.e., without the Lagrange multipliers and the velocity field obtained with the traction condition on the inlet and the outlet. All other variables (mesh, material parameters etc.) were kept fixed.

\subsection{Ionic Electrodiffusion in Cellular Geometries}

Many cerebral pathological conditions, e.g., spreading depression and epilepsy [46] are associated with changes in ion concentrations in the brain tissue. In this last example, we consider a model of ionic electrodiffusion in intracellular and extracellular domains, separated by a cell membrane. We represent the intracellular and extracellular domains as separate two-dimensional subdomains with the cell membrane as a topologically one-dimensional submesh. For more details of the mathematical model and numerical method, we refer to [17].

For this specific test case, illustrated in Figure 13, we consider a domain $\Omega \subset \mathbb{R}^{2}$ consisting of $\Omega_{i} \subset \mathbb{R}^{2}$ and $\Omega_{e} \subset \mathbb{R}^{2}$ representing the intracellular and extracellular spaces, respectively, together with the cell membrane $\Gamma=\bar{\Omega}_{i} \cap \bar{\Omega}_{e}$. The unknowns are the ion concentrations $[k]_{i}$ (resp. $[k]_{e}$ ) for each ion species $k \in K=\left\{\mathrm{Na}^{+}, \mathrm{K}^{+}, \mathrm{Cl}^{-}\right\}$, the electrical potential $\phi_{i}$ (resp. $\phi_{e}$ ) in the intracellular space $\Omega_{i}$ (resp. extracellular space $\Omega_{e}$ ), and the total ionic current density $I_{M}$ at the (lower dimensional) cell membrane $\Gamma$.

The evolution and distribution of the ion concentration $[k]_{r}, r=\{i, e\}$ for $k \in K$ is described by the continuity equation as

$$
\frac{\partial[k]_{r}}{\partial t}+\nabla \cdot J_{r}^{k}=f_{r} \text { in } \Omega_{r}
$$

The ion flux density $J_{r}^{k}(r=\{i, e\})$ is expressed in terms of the ion concentration gradients $\nabla[k]_{r}$ for $k \in K$ and the electrical potential gradients $\nabla \phi_{r}$ as

$$
J_{r}^{k}=-D_{r}^{k} \nabla[k]_{r}-\frac{D_{r}^{k} z^{k}}{\psi}[k]_{r} \nabla \phi_{r}
$$

where $D_{r}^{k}, r=\{i, e\}$ is the effective diffusion coefficient and $z^{k}$ the valence of the ion species $k \in K$. $\psi=R T F^{-1}$ with $F$ the Faraday's constant, $T$ is the absolute temperature, and $R$ the gas constant. Moreover, these ion flux densities are assumed to satisfy the electroneutrality assumption:

$$
F \sum_{k \in K} z^{k} \nabla \cdot J_{r}^{k}=0 \text { in } \Omega_{r} \forall r \in\{i, e\} .
$$

Assuming that no charge can leave or enter the system yields the exterior boundary condition

$$
F \sum_{k \in K} z^{k} J_{e}^{k} \cdot n_{e}=0 \text { on } \delta \Omega_{e} .
$$



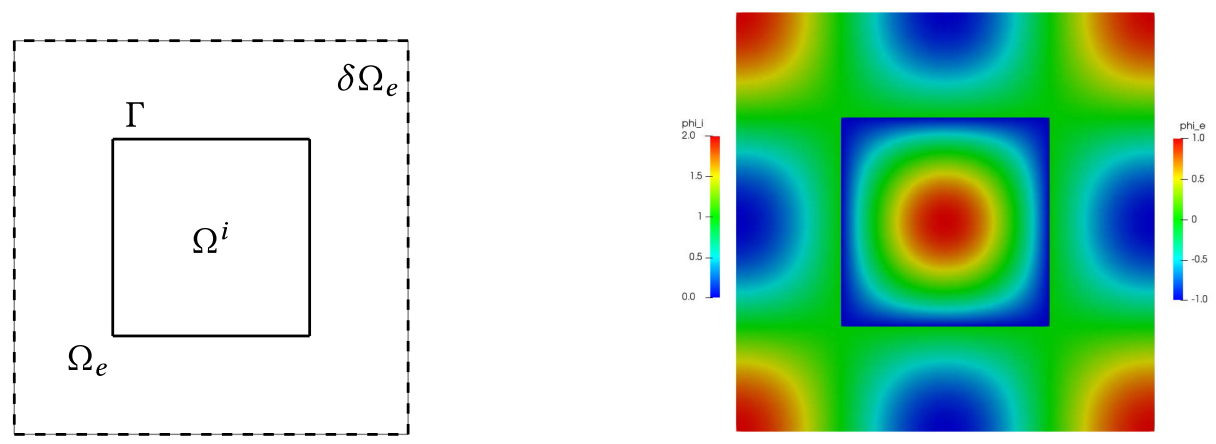

Fig. 13. Illustration of the domain composed of the extra cellular space $\Omega_{e}$, the intra cellular space $\Omega_{i}$ and the membrane $\Gamma$ as their interface (left). First order approximation of the potentials $\phi_{i} \in \Omega_{i}$ and $\phi_{e} \in \Omega_{e}$ matching the manufactured solution (49) (right).

The transmembrane potential is introduced as the difference between the intracellular and extracellular potential $\phi_{M}=\phi_{i}-\phi_{e}$ at the cell membrane, which satisfies

$$
\frac{\partial \phi_{M}}{\partial t}=\frac{1}{C_{M}}\left(I_{M}-I_{c h}\right),
$$

where $C_{M}$ and $I_{\mathrm{ch}}$ are the given capacitance and ion species specific channel current, respectively. This additional Equation (47) giving the total ionic current density $I_{M} \in \Gamma$ is coupled with (43) by the conservation equation of $I_{M}$ over the cell membrane as

$$
-F \sum_{k \in K} z^{k} J_{i}^{k} \cdot n_{i}=F \sum_{k \in K} z^{k} J_{e}^{k} \cdot n_{e} \equiv I_{M}
$$

We introduce manufactured solutions $[k]_{r}, k \in K$ for the ion concentrations and $\phi_{r}$ for the electric potential $(r=\{i, e\})$ satisfying (43)-(48) on $\Omega=\Omega_{i} \cup \Omega_{e}=[0,1] \times[0,1]$.

$$
\begin{aligned}
\mathrm{Na}_{i} & =0.7+0.3 \sin (2 \pi x) \sin (2 \pi y)\left(1+e^{-t}\right) \\
\mathrm{Na}_{e} & =1.0+0.6 \sin (2 \pi x) \sin (2 \pi y)\left(1+e^{-t}\right) \\
\mathrm{K}_{i} & =0.3+0.3 \sin (2 \pi x) \sin (2 \pi y)\left(1+e^{-t}\right) \\
\mathrm{K}_{e} & =1.0+0.2 \sin (2 \pi x) \sin (2 \pi y)\left(1+e^{-t}\right) \\
\mathrm{Cl}_{i} & =1.0+0.6 \sin (2 \pi x) \sin (2 \pi y)\left(1+e^{-t}\right)^{\prime} \\
\mathrm{Cl}_{e} & =2.0+0.8 \sin (2 \pi x) \sin (2 \pi y)\left(1+e^{-t}\right) \\
\phi_{i} & =\cos (2 \pi x) \cos (2 \pi y)\left(1+e^{-t}\right) \\
\phi_{e} & =\cos (2 \pi x) \cos (2 \pi y)
\end{aligned}
$$

As our test case here, we then consider the boundary conditions and sources induced by inserting the manufactured solution (49) into Equations (43)-(48).

We define a uniform mesh $\mathcal{T}^{1}$ of the unit square $\Omega$, from which we build two two-dimensional meshes $\mathcal{T}^{2}$ and $\mathcal{T}^{3}$ relative to the intracellular domain $\Omega_{i}$ and the extracellular domain $\Omega_{e}$, respectively. We consider a lower-dimensional mesh $\mathcal{T}^{4}$ to define the cell membrane $\Gamma$. All the unknowns in our system i.e., the ion concentrations $[k]_{r}, r=\{i, e\}, k=\left\{\mathrm{Na}^{+}, \mathrm{K}^{+}, \mathrm{Cl}^{-}\right\}$and the electrical potentials $\phi_{i}$ and $\phi_{e}$, are approximated using piecewise continuous elements of order $l \geq 1$.

The corresponding solutions for the electrical potentials $\phi_{i}$ and $\phi_{e}$ are shown in Figure 13. A convergence study of the error of the approximation in the $L_{2}$ and $H_{1}$ norms for the Sodium $\left(\mathrm{Na}^{+}\right)$ 


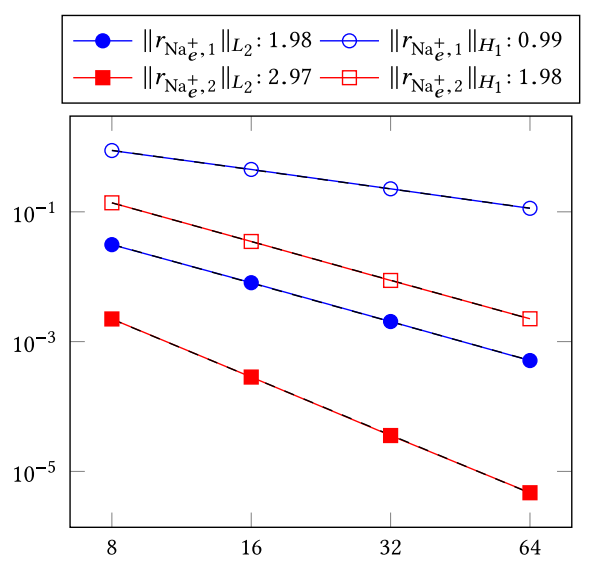

(a) Convergence study $-\mathrm{Na}_{e}^{+}$

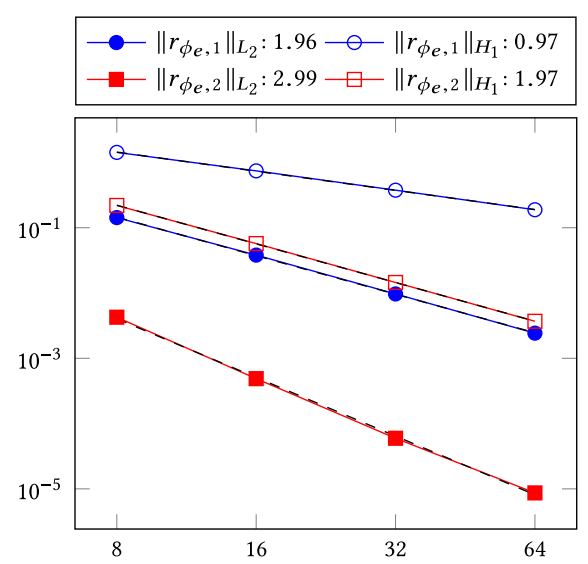

(c) Convergence study - $\phi_{e}$

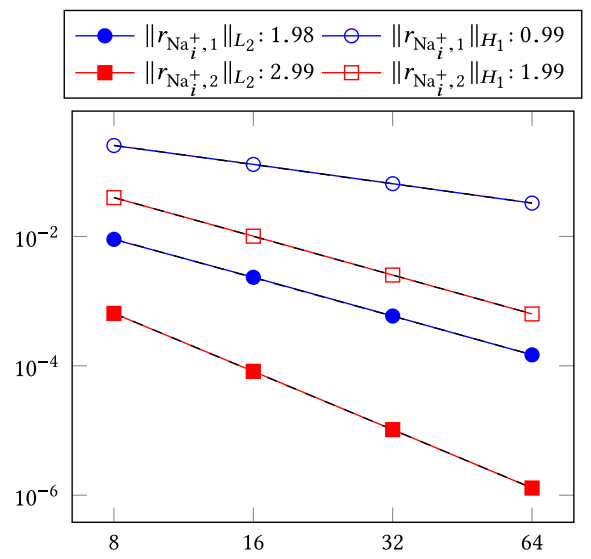

(b) Convergence study $-\mathrm{Na}_{i}^{+}$

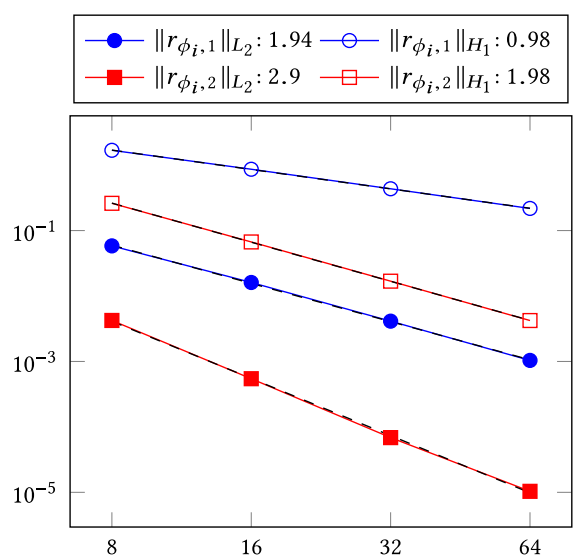

(d) Convergence study - $\phi_{i}$

Fig. 14. Convergence study for the KNP-EMI model given by Equations (43)-(48) based on the manufactured solution (49). The plots show the approximation error $\left\|e_{h}\right\|_{Q}$ (37) in the $Q$ norm depending on the mesh resolution. $\left\|r_{v, l}\right\|_{Q}$ defines the rate of convergence for the solution variable $v$ in the $Q$ norm with approximation order $l$. The resulting convergence rates are given in legend. The expected convergence rates in $L_{2}$ and $H_{1}$ norms are obtained for both first- and second order approximations of sodium $\left(\mathrm{Na}^{+}\right)$concentration $(\mathrm{a}$ and b) and for the electric potentials $\phi_{e}(\mathrm{c})$ and $\phi_{i}(\mathrm{~d})$.

concentrations and electrical potentials $\phi_{i}, \phi_{e}$ is shown in Figure 14. The convergence rates indicate that we obtain an expected order of convergence (37) for both polynomial orders $l=\{1,2\}$. The analogous results are obtained for the other ion concentrations.

\section{DISCUSSION AND LIMITATIONS}

\subsection{Mesh Generations and Remeshing}

Our implementation is limited to one generation of meshes, i.e., we only support parent-child and sibling meshes. As such, nested mesh representations, i.e., submeshes of submeshes are not supported, and such constructions should instead be unrolled to a list of child-parent meshes. This 
limitation is in part associated with the restriction to the codimension-one case: lower-dimensional submeshes of lower-dimensional submeshes would easily involve coupling between meshes with a higher dimensional gap.

There also exists the problem associated with domain decomposition and load balancing for related meshes, when running a parallel simulation. For example, in a typical problem with a parent volume mesh and a child mesh defined on the surface facets, the child mesh may have no nodes at all on some processes (some processes may have no surface facets). It might seem reasonable to redistribute the child mesh to improve the load balance. However, this would result in extra communication when coupling between the two meshes, as their related cells would no longer be on the same process. The advantages of repartitioning are likely to be highly problem specific, and depend on the exact nature of the relationship between the two meshes, how much coupling there is between them and the relative size of the problem on each mesh. For example, the number of degrees of freedom on the surface is usually smaller than in the volume mesh. The additional complexity, and problem specificity seems to preclude a generic solution. It may be possible to use the connectivity of the combined parent-child meshes as input to the domain decomposition routines, but this is as yet untested.

\subsection{Choice of Symbolic Representation for Mixed Function Spaces}

In the approach and implementation presented here, we have introduced light-weight language abstractions for mixed function spaces and arguments and coefficients defined over such. In particular, mixed function spaces over mixed meshes and the associated fields are represented simply as tuples of function spaces and fields, each defined over a single mesh. Clearly, there are advantages and disadvantages to this approach. We consider the light-weight structure and ease of working with separate blocks advantageous in terms of simplicity in design and efficiency in implementation, in particular for multi-physics use cases. In this regard, mixed spaces differs from mixed elements; e.g., for the latter the complete local element tensor is computed for all components in one go. We consider the choice of allowing for block-by-block computations to be more flexible and potentially more efficient. Similar structures have also been used by related implementations such as by fenics_ii [27], and multiphenics [5]. On the other hand, a MixedFunctionspace is not a Functionspace, and thus a Function cannot be defined directly on a MixedFunctionspace. Thus, for instance, symbolic derivatives with regard to state variables must be defined block by block. To address this disadvantage, one could consider extending the MixedFunctionspace symbolics in the UFL layer, while maintaining a block-wise representation in the code generation and linear algebra layers.

\subsection{Higher Codimensions}

While we have focused on codimension one submeshes in this article, many of the concepts, abstractions and algorithms extend to higher dimensional gaps. We briefly discuss such extensions for the components in introduced in Section 2.6 to codimension two cases below.

Mappings for nested submeshes. The cells of a submesh of codimension $e \geq 1$ are mesh entities of codimension $e$ in the parent mesh $\mathcal{T}$. For example, in the important case of codimension $e=2$ and $d=3$, each cell in the submesh is an edge in the parent mesh, extending (24) to

$$
\mathcal{M}_{v}^{i}: \mathcal{V}^{i} \rightarrow \mathcal{V} \text { and } \mathcal{M}^{i}: C^{i} \rightarrow C, \mathcal{F} \text { or } \mathcal{E},
$$

with $\mathcal{E}$ the set of edges in $\mathcal{T}$. The computation of $\mathcal{M}^{i, j}: C^{i} \longrightarrow \mathcal{E}^{j}$ naturally follows the approach introduced for the codimension-one case (see, e.g., Figure 4). The mapping $\mathcal{M}^{i}: C^{i} \rightarrow \mathcal{E}$ finds the edge index $e_{k} \in \mathcal{S}(\mathcal{T})$ associated with the lower dimensional cell index $c_{k} \in \mathcal{T}^{i}$. The mesh 
connectivity $(d-2) \rightarrow d$ then gives the indices of the cells sharing this edge in $\mathcal{S}(\mathcal{T})$, and the inverse mapping $\mathcal{M}^{j}$ is used to determine the corresponding set of cells in $\mathcal{T}^{j}$. The edge of index $e_{k} \in \mathcal{T}$ is shared by an arbitrary number of cells $n_{s}$ in $\mathcal{T}$ that might not all be included in $\mathcal{T}^{j}$ i.e., $n_{s} \geqslant n_{s}^{j}$. The subset of cells in $\mathcal{T}^{j}$ of size $n_{s}^{j} \geqslant 1$ is enough to find the index of edge in $\mathcal{E}^{j}$ relative to the lower dimensional cell $c_{k} \in \mathcal{T}^{i}$.

Local element tensors computation. As described in Section 7.1, the computation of codimension one local element tensors mimics the previous exterior facet tensor implementation. The case of higher codimension such as 3D-1D local element tensors entails integration over edges, which is lacking in the current version of FEniCS. We imagine that the implementation of this feature requires both new abstractions in the language, i.e., a new integral type dedicated to edges together with the corresponding measure (see Section 4.2), and new geometrical ingredients involved in the transformation from reference simplices of lower dimension $d-2$ to real elements of dimension $d$. Note that the scope of this feature is not limited to 3D-1D couplings but that it is also relevant for monodomain problems involving edge boundary conditions for instance. The computation of edge local element tensors then needs its dedicated tabulate_tensor function based on edge integration.

Assembly. Assuming the availability of the appropriate mesh mappings and local element tensors described above, the revised assembly algorithm presented in Section 6 can be directly extended to higher dimensional gaps, iterating over the star $S_{K}$ composed of an arbitrary number of elements in Algorithm 2.

\subsection{Handling Discontinuities and Non-Single-Valued Traces}

Our discussion up to now has assumed that the relevant traces have been single-valued and welldefined. For discontinuous finite element spaces, the traces on lower-dimensional entities are typically not single-valued. FEniCS traditionally handles such traces, e.g., in the context of discontinuous Galerkin finite element methods, by explicit restrictions. More precisely, in UFL [2] the (two) cells sharing an interior facet are labeled by + and - and the discontinuous functions involved in the variational form must explicitly be restricted when integrating over interior facets. For instance, the jump $\llbracket u \rrbracket$ of a (discontinuous) function $u$ over an interior facet can be expressed as the difference $\llbracket u \rrbracket=u^{+}-u^{-}$where $u^{+}$and $u^{-}$represent the function values from both sides of the considered facet.

Mixed dimensional problems with an integration domain of lower dimension (i.e., with dimension $\left.d^{\mathcal{K}}<\max \left(d^{1}, d^{2}\right)\right)$ involve local element tensors over the cells in the star $S_{K}$, as described in Section 6. In our approach, these tensors are computed as exterior facet element tensors and combined into the global matrix. By default, the exterior facet tensor computation considers the function value from the ${ }^{\prime}{ }^{\prime}$ side for each shared cell, while the ${ }^{\prime}{ }^{\prime}$ side is ignored. For discontinuous spaces, where the trace of the integrand is not single-valued, this choice can give ambiguous behaviour. We therefore consider these cases to not be fully supported in the presented pipeline. The extension of the framework to coupling discontinuous traces across submeshes would require changes to the mixed assembly algorithm for handling restrictions. In particular, the zeroing step in Algorithm 2 needs to be revised to, e.g., mimic interior facet assembly when restrictions are specified. We emphasize however that diagonal (sub)blocks with interior facet integrals, i.e., forms involving arguments over a single mesh, are still supported.

\section{CONCLUSIONS}

We have introduced a set of abstractions, algorithms, and design guidelines for the automated assembly and solution of mixed domain and mixed dimensional finite element methods. Further, 
we have presented a realization of these general concepts within the FEniCS finite element software. These features thus allow for the solution of PDEs posed on different domains, either of the same dimension or involving codimension one subdomains. We have illustrated the features with a number of numerical examples starting from a basic constrained Poisson problem to a nontrivial model of ionic electrodiffusion. However, we argue that this series of examples only begin to illustrate the possibilities offered by the framework. Future work will focus on the extension of the framework to coupled problems with higher codimensions, non-conforming meshes, and optimal mesh partitioning.

\section{ACKNOWLEDGMENTS}

We thank Martin Alnæs, Miroslav Kuchta and Jørgen Dokken (Simula Research Laboratory) for constructive discussion on topics related to the manuscript.

\section{REFERENCES}

[1] Martin S. Alnæs, Jan Blechta, Johan Hake, August Johansson, Benjamin Kehlet, Anders Logg, Chris Richardson, Johannes Ring, Marie E. Rognes, and Garth N. Wells. 2015. The FEniCS project version 1.5. Archive of Numerical Software 3, 100 (2015), 9-23. DOI : https://doi.org/10.11588/ans.2015.100.20553

[2] Martin S. Alnæs, Anders Logg, Kristian B. Ølgaard, Marie E. Rognes, and Garth N. Wells. 2014. Unified form language: A domain-specific language for weak formulations of partial differential equations. ACM Transactions on Mathmatical Software 40, 2, (Mar. 2014), 1-37. DOI : https://doi.org/10.1145/2566630

[3] Douglas N. Arnold, Franco Brezzi, Bernardo Cockburn, and Donatella Marini. 2000. Discontinuous galerkin methods for elliptic problems. In Discontinuous Galerkin Methods. Bernardo Cockburn, George E. Karniadakis, and Chi-Wang Shu (Eds.), Springer, Berlin, 89-101.

[4] Satish Balay, Shrirang Abhyankar, Mark F. Adams, Jed Brown, Peter Brune, Kris Buschelman, Lisandro Dalcin, Victor Eijkhout, William D. Gropp, Dinesh Kaushik, Matthew G. Knepley, Dave A. May, Lois Curfman McInnes, Richard Tran Mills, Todd Munson, Karl Rupp, Patrick Sanan, Barry F. Smith, Stefano Zampini, Hong Zhang, and Hong Zhang. 2018 PETSc Web page. Retrieved from http://www.mcs.anl.gov/petsc. http://www.mcs.anl.gov/petsc.

[5] F. Ballarin. 2016. Multiphenics - Easy Prototyping of Multiphysics Problems in FEniCS. Retrieved October 10, 2016 from https://mathlab.sissa.it/multiphenics.

[6] W. Bangerth, R. Hartmann, and G. Kanschat. 2007. Deal.II -A general purpose object oriented finite element library. ACM Transactions on Mathmatical Software 33, 4 (2007), 1-27.

[7] S. Bertoluzza, V. Chabannes, C. Prud'Homme, and M. Szopos. 2017. Boundary conditions involving pressure for the Stokes problem and applications in computational hemodynamics. Computer Methods in Applied Mechanics and Engineering 322 (2017), 58-80. DOI : https://doi.org/10.1016/j.cma.2017.04.024

[8] Wietse Marijn Boon. 2018. Conforming Discretizations of Mixed-Dimensional Partial Differential Equations. Doctoral thesis. University of Bergen, Bergen, Norway. Retrieved from http://bora.uib.no/handle/1956/18159.

[9] Wietse M. Boon, Jan M. Nordbotten, and Jon E. Vatne. 2021. Functional Analysis and Exterior Calculus on MixedDimensional Geometries. Annali di Matematica Pura ed Applicata (1923-) 200, 2 (2021), 757-789.

[10] S. Brenner and R. Scott. 2007. The Mathematical Theory of Finite Element Methods. Springer. Retrieved from https: //books.google.no/books?id=ci4c_R0WKYYC.

[11] Franco Brezzi, Jim Douglas, and L. Donatella Marini. 1985. Two families of mixed finite elements for second order elliptic problems. Numerische Mathemtik. 47, 2 (1985), 217-235.

[12] Erik Burman, Susanne Claus, Peter Hansbo, Mats G. Larson, and André Massing. 2015. CutFEM: Discretizing geometry and partial differential equations. International fournal for Numerical. Methods in Engineering 104, 7 (2015), 472-501. DOI : https://doi.org/10.1002/nme.4823

[13] Sunčica Čanić, Marija Galić, Matko Ljulj, Boris Muha, Josip Tambača, and Yifan Wang. 2019. Analysis of a linear 3D fluid-mesh-shell interaction problem. Zeitschrift Für Angewandte Mathematik und Physik 70, 2 (Feb. 2019 ), 44. DOI : https://doi.org/10.1007/s00033-019-1087-1

[14] P. G. Ciarlet. 1976. Numerical Analysis of the Finite Element Method. Les Presses de l.Université de Montréal, Series "Séminaire de Mathématiques Supérieures" 59 (1976).

[15] P. G. Ciarlet. 2002. The Finite Element Method for Elliptic Problems. Society for Industrial and Applied Mathematics. Retrieved from https://books.google.no/books?id=isEEyUXW9qkC.

[16] Cécile Daversin-Catty, Chris N. Richardson, Ada J. Ellingsrud, and Marie E. Rognes. 2019. Mixed-dimensionalexamples v2019.1. Retrieved from https://zenodo.org/record/3525001. 
[17] A. J. Ellingsrud, A. Solbrå, G. T. Einevoll, G. Halnes, and M. E. Rognes. 2020. Finite element simulation of ionic electrodiffusion in cellular geometries. Frontiers in Neuroinformatics 14 (2020), 11.

[18] A. Ern and J. L. Guermond. 2004. Theory and Practice of Finite Elements. Springer. Retrieved from https://books.google. no/books?id=CCjm79FbJbcC.

[19] Patrick E. Farrell, David A. Ham, Simon W. Funke, and Marie E. Rognes. 2013. Automated derivation of the adjoint of high-level transient finite element programs. SIAM fournal on Scientific Computing 35, 4 (2013), C369-C393.

[20] F. Hecht. 2012. New development in FreeFem++. Journal of Numerical Mathematics 20, 3-4 (2012), 251-265.

[21] Luca Heltai and Francesco Costanzo. 2012. Variational implementation of immersed finite element methods. Computer Methods in Applied Mechanics and Engineering 229-232 (2012), 110-127. DOI : https://doi.org/10.1016/j.cma.2012.04.001

[22] Miklós Homolya, Lawrence Mitchell, Fabio Luporini, and David A. Ham. 2018. TSFC: A structure-preserving form compiler. SIAM Journal on Scientific Computing 40, 3 (2018), C401-C428. DOI : https://doi.org/10.1137/17M1130642

[23] August Johansson, Benjamin Kehlet, Mats G. Larson, and Anders Logg. 2019. Multimesh finite element methods: Solving PDEs on multiple intersecting meshes. Computer Methods in Applied Mechanics and Engineering 343 (2019), 672-689. DOI : https://doi.org/10.1016/j.cma.2018.09.009

[24] George Karypis. 2011. METIS and ParMETIS. In Encyclopedia of Parallel Computing. Springer, 1117-1124. DOI : https: //doi.org/10.1007/978-0-387-09766-4_500

[25] Eirik Keilegavlen, Runar Berge, Alessio Fumagalli, Michele Starnoni, Ivar Stefansson, Jhabriel Varela, and Inga Berre. 2021. Porepy: An open-source software for simulation of multiphysics processes in fractured porous media. Computational Geosciences 25, 1 (2021), 243-265.

[26] Timo Koch, Katharina Heck, Natalie Schröder, Holger Class, and Rainer Helmig. 2018. A new simulation framework for soil-root interaction, evaporation, root growth, and solute transport. Vadose Zone fournal 17, 1 (2018). DOI : https: //doi.org/10.2136/vzj2017.12.0210

[27] Miroslav Kuchta. 2019. Assembly of multiscale linear PDE operators. In Numerical Mathematics and Advanced Applications ENUMATH. F. J. Vermolen and C. Vuik (Eds.), Springer, Cham, 641-650.

[28] Miroslav Kuchta, Magne Nordaas, Joris C. G. Verschaeve, Mikael Mortensen, and Kent-Andre Mardal. 2016. Preconditioners for saddle point systems with trace constraints coupling 2D and 1D domains. SIAM fournal on Scientific Computing 38, 6 (2016), B962-B987. DOI : https://doi.org/10.1137/15M1052822

[29] Martin Werner Licht. 2017. Complexes of discrete distributional differential forms and their homology theory. Foundations of Computational Mathematics 17, 4 (Aug. 2017), 1085-1122. DOI : https://doi.org/10.1007/s10208-016-9315-y

[30] A. Logg. 2009. Efficient representation of computational meshes. International fournal of Computational Science and Engineering 4, 4 (Nov. 2009), 283-295. DOI: https://doi.org/10.1504/IJCSE.2009.029164

[31] Anders Logg, Kent-Andre Mardal, Garth N. Wells, et al. 2012. Automated Solution of Differential Equations by the Finite Element Method. Springer, Berlin. DOI : https://doi.org/10.1007/978-3-642-23099-8

[32] Anders Logg, Kristian B. Ølgaard, Marie E. Rognes, and Garth N. Wells. 2012. FFC: The FEniCS Form Compiler. Springer, Berlin. 227-238.

[33] Anders Logg and Garth N. Wells. 2010. DOLFIN: Automated finite element computing. ACM Transactions on Mathematical Software 37, 2, (Apr. 2010), 1-28. DOI : https://doi.org/10.1145/1731022.1731030

[34] Anders Logg, Garth N. Wells, and Johan Hake. 2012. DOLFIN: a C++/Python Finite Element Library. Springer, Berlin, $173-225$.

[35] J. -C. Nédélec. 1980. Mixed finite elements in $\mathbb{R}^{3}$. Numerische Mathematik 35, 3 (1980), 315-341.

[36] J. -C. Nédélec. 1986. A new family of mixed finite elements in $\mathbb{R}^{3}$. Numerische Mathematik 50, 1 (1986), 57-81.

[37] Kristian B. Ølgaard, Anders Logg, and Garth N. Wells. 2008. Automated code generation for discontinuous galerkin methods. SIAM fournal on Scientific Computing 31, 2 (Nov. 2008), 849-864. DOI : https://doi.org/10.1137/070710032

[38] François Pellegrini and Jean Roman. 1996. Scotch: A software package for static mapping by dual recursive bipartitioning of process and architecture graphs. In High-Performance Computing and Networking. H. Liddell, A. Colbrook, B. Hertzberger, and P. Sloot (Eds.), Springer, Berlin, 493-498.

[39] Christophe Prud'Homme, Vincent Chabannes, Vincent Doyeux, Mourad Ismail, Abdoulaye Samake, and Gonçalo Pena. 2012. Feel++: A computational framework for galerkin methods and advanced numerical methods. ESAIM: Proceedings 38 (Dec. 2012), 429-455. DOI : https://doi.org/10.1051/proc/201238024

[40] Florian Rathgeber, David A. Ham, Lawrence Mitchell, Michael Lange, Fabio Luporini, Andrew T. T. Mcrae, GheorgheTeodor Bercea, Graham R. Markall, and Paul H. J. Kelly. 2016. Firedrake: Automating the finite element method by composing abstractions. ACM Transactions on Mathematical Software 43, 3, Article 24 (Dec. 2016), 27 pages. DOI : https: //doi.org/10.1145/2998441

[41] P. -A. Raviart and J. M. Thomas. 1977. A mixed finite element method for 2nd order elliptic problems. In Mathematical Aspects of Finite Element Methods (Proc. Conf., Consiglio Naz. delle Ricerche (C.N.R.), Rome, 1975). Lecture Notes in Math., Vol. 606, Springer, Berlin, 292-315. 
[42] M. E. Rognes, D. A. Ham, C. J. Cotter, and A. T. T. McRae. 2013. Automating the solution of PDEs on the sphere and other manifolds in FEniCS 1.2. Geoscientific Model Development 6, 6 (2013), 2099-2119. DOI : https://doi.org/10.5194/ gmd-6-2099-2013

[43] Abdoulaye Samake. 2014. Large Scale Nonconforming Domain Decomposition Methods. Theses. Université Grenoble Alpes, Saint-Martin-d'Hères, France. Retrieved from https://tel.archives-ouvertes.fr/tel-01092968.

[44] J. Schöberl. 2019. NGSolve Finite Element Library. Retrieved from https://sourceforge.net/projects/ngsolve/.

[45] Nicolas Schwenck, Bernd Flemisch, Rainer Helmig, and Barbara I. Wohlmuth. 2015. Dimensionally reduced flow models in fractured porous media: Crossings and boundaries. Computational Geosciences 19, 6 (Dec. 2015), 1219-1230. DOI : https://doi.org/10.1007/s10596-015-9536-1

[46] George G. Somjen. 2001. Mechanisms of spreading depression and hypoxic spreading depression-like depolarization. Physiological Reviews 81, 3 (2001), 1065-1096. DOI : https://doi.org/10.1152/physrev.2001.81.3.1065 PMID: 11427692.

[47] Marcela Szopos. 2017. Mathematical Modeling, Analysis and Simulations for Fluid Mechanics and Their Relevance to in Silico Medicine. Habilitation à diriger des recherches. Université de Strasbourg, IRMA UMR 7501. Retrieved from https://tel.archives-ouvertes.fr/tel-01646867.

[48] Aslak Tveito, Karoline H. Jæger, Miroslav Kuchta, Kent-Andre Mardal, and Marie E. Rognes. 2017. A cell-based framework for numerical modeling of electrical conduction in cardiac tissue. Frontiers in Physics 5 (2017), 48. DOI : https: //doi.org/10.3389/fphy.2017.00048

[49] Jun Zhou, Wan Kan Chan, and Justin Schwartz. 2019. Modeling of Quench Behavior of $\mathrm{YBa}_{2} \mathrm{Cu}_{3} \mathrm{O}_{7-\delta}$ pancake magnets and distributed temperature sensing-based quench detection for operating temperature $30 \mathrm{~K}-77 \mathrm{~K}$. IEEE Transactions on Applied Superconductivity 29, 1 (2019), 1-11. DOI : https://doi.org/10.1109/TASC.2018.2874423

Received November 2019; revised February 2021; accepted June 2021 